\title{
Annotationes Epigraphicae VI \\ Zu einigen Inschriften aus den römischen Provinzen Germania inferior und Germania superior*
}

\author{
Andreas Kakoschke
}

I.

Im Jahre 2004 fand sich in der Bechergasse in Köln/CCAA die rechte Hälfte eines kleinen Altars (Höhe $46 \mathrm{~cm}$ - Breite $10 \mathrm{~cm}$ - Tiefe 26,5 cm) aus Kalkstein (Abb.1). Das Fragment besteht aus zwei Teilen mit einer vierzeiligen Inschrift, die noch Spuren einer roten Ausmalung aufweist. B. und H. GALSTERER, die den Altar in das Ende des ersten bzw. in den Anfang des zweiten Jahrhunderts n. Chr. datieren, geben den Text des Steins in Umschrift wie folgt wieder: ${ }^{1}$

$$
\begin{aligned}
& {[I(\text { ovi }) \cdot O(\text { ptimo } \bullet] M(\text { aximo })} \\
& {[\text { ca. } 3] \operatorname{VIP}} \\
& {[\text { ca. } 3] R V S} \\
& {[v(\text { otum }) s(\text { olvit })] l(\text { ibens }) m(\text { erito })}
\end{aligned}
$$

Dabei erblicken die Ersteditoren in VIP (Zeile 2) den Teil eines Gentilnomens. ${ }^{2}$ Bei RVS (Zeile 3) soll es sich um den Rest eines Cognomens handeln, für das B. und H. GALSTERER eine Ergänzung zu den Namen Clarus oder Hilarus vorschlagen.

Statt VIP scheint allerdings eher die Lesung VLP zuzutreffen. Die nur schwach ausgeprägte waagrechte Haste des L reicht dabei unter die senkrechte Haste des folgenden P. Offenbar war der Steinmetz am Ende der Zeile in Platznot geraten und musste die Buchstaben L und P daher eng aneinander rücken. Diese Auffassung wird auch durch B. und H. GALSTERER bestätigt, die den unteren Teil des Altars in

\footnotetext{
* Für die Bereitstellung einiger Photos danke ich Herrn Prof. Dr. R. Wiegels und Herrn PD Dr. K. Matijević (CIL XIII/2-Projekt, Universität Trier). Herrn Dr. C. Wenzel (Archäologisches Museum Frankfurt am Main) schulde ich Dank für die Informationen zum Bronzetäfelchen aus Heddernheim/Nida. Bei Herrn Prof. Dr. R. Nedoma bedanke ich mich für Hinweise zur Weihinschrift des Primio Cellissi fil. aus Köln/CCAA. Für Unterstützung im technischen Bereich danke ich Herrn Ch. Lindner (Osnabrück). - Ligaturen bzw. Nexus werden im Folgenden durch unterstrichene Buchstaben wiedergegeben. - Die Annotationes Epigraphicae I und II erschienen in der FeRA 26 und 27 (2015), 10-26 bzw. 20-42. Die Annotationes Epigraphicae III erschienen im GFA 19 (2015), 179-197, die Annotationes Epigraphicae IV in der FeRA 29 (2016), 43-76 und die Annotationes Epigraphicae V in der FeRA 30 (2016), 1-25.

${ }^{1}$ IKöln ${ }^{2}$ 528-529. Nr.791 = AE 2010, 1017 = EDCS Nr.47700079 = EDH Nr.HD067451 (jeweils mit identischer Lesung). - Der Stein befindet sich heute im Römisch-Germanischen Museum in Köln (Inv.Nr.F.B.2004.23,45-1242).

${ }^{2}$ Mit VIP beginnende Gentilnomina sind abgesehen von Vipsanius recht selten (SOLIN/SALOMIES 1994, 209, KAKOSCHKE 2006, 439-440). In Niedergermanien finden sich Belege für folgende Namen: Viponius [2. N. 115. Nr.241 (bei Benzelrath)], Vipsanius [CIL XIII 8572 (Gripswald bei KrefeldGellep/Gelduba)]. Entsprechendes gilt für mit VIP beginnende Cognomina (SoLIN/SALOMIES 1994, 423, KAKOSCHKE 2008, 463). In Niedergermanien ist nur ein mit VIP beginnender Name bezeugt: Viperinus [CIL XIII 8353 (Köln/CCAA)]. Ferner sind Namen mit der Buchstabengruppe VIP innerhalb des Namens äußerst selten [s. z.B. EDR Nr.100992 (Rom) mit dem Namen Heuippa]. Verlässt man das Feld der Namen, passt VIP lediglich noch zu dem Begriff sesquiplicarius.
} 
dem Sammelwerk „Die römischen Steininschriften aus Köln“ fehlerhafterweise ein zweites Mal mit entsprechender Lesung aufgenommen haben. ${ }^{3}$

VLP ist wohl nicht zu [al(a) SJulp(icia) oder ähnlich zu ergänzen. Die Reitereinheit war seit flavischer Zeit in Niedergermanien, offenbar im Raum Köln/CCAA, stationiert. Mehrere Inschriften aus Köln/CCAA erwähnen Reiter der Truppe. ${ }^{4}$ Die Kürze der Inschrift und das in Zeile $3 \mathrm{zu}$ vermutende Cognomen schließen die genannte Ergänzung in Zeile 2 wohl aus. Jedoch könnte die Buchstabengruppe zu dem Gentilnomen [S] ulp (icius) ergänzt werden. ${ }^{5}$ Wahrscheinlicher ist m.E. allerdings die Lesung Ulp(ius). Am Anfang der Zeile stand dann mit einiger Sicherheit das abgekürzte Pränomen Marcus (Abb.2). Angesichts der wohl zutreffenden Ergänzung der ersten und letzten Zeile durch B. und H. GALSTERER bieten das M und ein Wortrenner auch die nötige Breite, um die zweite Zeile ganz zu füllen. Zudem steht die Ergänzung im Einklang mit der Datierung des Fragments. ${ }^{6}$

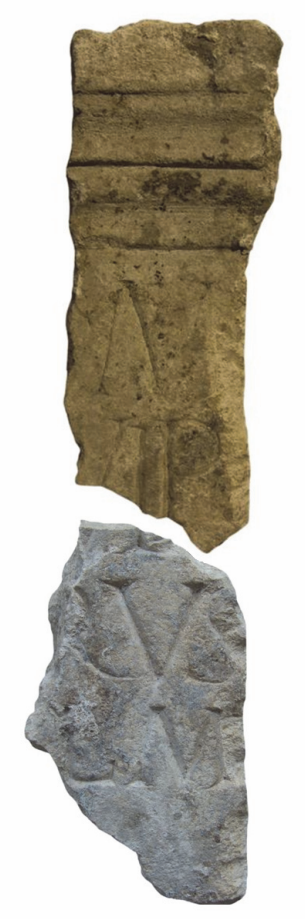

Abb.1: Fragmentarische Weihinschrift aus Köln/CCAA: AE 2010, 1017

[C Römisch-Germanisches Museum, Köln (Photomontage A.K.)]

In der dritten Zeile scheint eine Ergänzung zu den von B. und H. GALSTERER vorgeschlagenen Cognomina Clarus oder Hilarus aus Platzgründen zweifelhaft. Entsprechendes gilt für das etwas schmalere gängige Carus. Daher wird man sich eher für das nicht nur im Gebiet der CCAA vielfach bezeugte Verus entscheiden. Sicherheit ist jedoch nicht zu gewinnen. ${ }^{7}$

\footnotetext{
${ }^{3}$ IKöln $^{2}$ 209. Nr.246 = AE 2010, $1012=$ EDCS Nr.47600173 = EDH Nr.HD067442 (jeweils mit identischer Lesung). Für das Fragment mit der Inv.-Nr.F.B.2004.23,45-1228 geben B. und H. GALSTERER die Fundortangabe „Heumarkt, beim Bau der Tiefgarage“.

${ }^{4}$ CIL XIII $8185=$ IKöln ${ }^{2}$ 67-68. Nr.46, $8311=$ IKöln ${ }^{2}$ 305-306. Nr.365, $8312=$ IKöln ${ }^{2}$ 307. Nr.366. Zur ala Sulpicia civium Romanorum s. Alföldy 1968, 34-36, SPAUl 1994, 209.

${ }^{5}$ Zu Sulpicus s. KAKOSCHKE 2006, 383-384. GN 1242.

${ }^{6}$ Zu Ulpius s. KAKOSCHKE 2006, 404-406. GN 1329.

${ }^{7}$ Zu Verus s. KAKOSCHKE 2008, 445-446. CN 3295.
} 
Der Text der fragmentarischen Inschrift aus Köln/CCAA könnte also wie folgt gelautet haben:

$$
\begin{aligned}
& {[I(\text { ovi }) \cdot O(\text { ptimo }] \bullet M(\text { aximo })} \\
& {[M(\text { arcus }) \cdot] \text { Vlp }(\text { ius })} \\
& {[V e ?] r u s} \\
& {[v(\text { otum }) s(\text { olvit })] l(\text { ibens }) m(\text { erito })}
\end{aligned}
$$

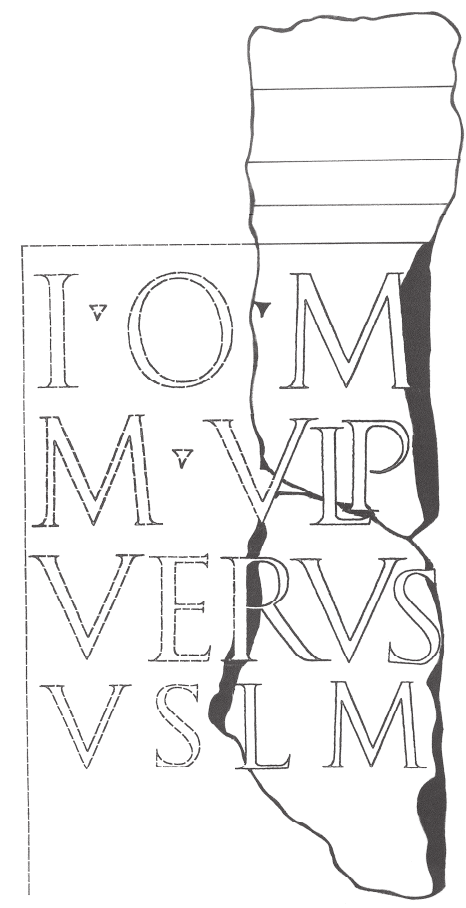

Abb.2: Leicht idealisierte Rekonstruktionszeichnung der Weihinschrift AE 2010, 1017 aus Köln/CCAA (Zeichnung A.K.)

\section{II.}

Ebenfalls in Köln/CCAA, in St. Kunibert, entdeckte man im Jahre 1978 die stark beschädigte linke Seite eines Altars (Höhe $65 \mathrm{~cm}$ - Breite $15 \mathrm{~cm}$ - Tiefe $13 \mathrm{~cm}$ ) aus Sandstein. Im oberen Teil des Fragments ist ein Teil der Altarbekrönung erhalten und unten ein Teil der Unterkante. Im unteren Teil erkennt man ferner noch die linke Seitenkante des Steins (Abb.3). B. und H. GALSTERER, die den Stein in das Ende des zweiten bzw. den Anfang des dritten Jahrhunderts n. Chr. datieren, ergänzen den Text der Inschrift wie folgt: ${ }^{8}$

$$
\begin{array}{ll} 
& {[\text { Ma]tronis }} \\
& {[M] \operatorname{ar}[\text { sacis?] }} \\
& \text { Suc[cessius?] } \\
& \text { Cri[spinus } ?] \\
5 \quad[\text { (otum) } s(\text { olvit }) ?]
\end{array}
$$

\footnotetext{
${ }^{8}$ IKöln $^{2}$ 144. Nr.150 = THOMAS 2014, 147. Nr.50 = EDCS Nr.47600192 (mit identischer Lesung). Der Stein befindet sich heute im Römisch-Germanischen Museum in Köln (Inv.-Nr.78,213a).
} 


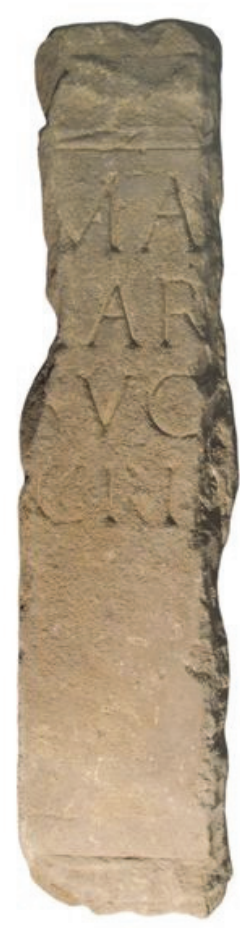

Abb.3: Fragmentarische Weihinschrift aus Köln/CCAA: IKöln ${ }^{2} 144$. Nr.150

(C) Römisch-Germanisches Museum, Köln)

Diese Lesung kann so jedoch nicht zutreffen, da für ein $\mathrm{M}$ am Beginn von Zeile 2 nicht genügend Raum zur Verfügung steht. ${ }^{9}$ Wie eine Rekonstruktionszeichnung (Abb.4) zeigt, ist wohl Ar[sacis] zu ergänzen, zumal am Beginn der Zeile kein Buchstabenrest auszumachen ist. ${ }^{10}$ Stimmt die Ergänzung der ersten und dritten Zeile zu Ma[tronis], ${ }^{11}$ mit einer oft belegten NI-Ligatur, bzw. zu dem hier favorisierten Suc [cessus], ${ }^{12}$ zeigt sich, dass der Steinmetz den Matronenbeinamen zentriert eingeschlagen hat. Größere Unsicherheit birgt lediglich die letzte Zeile. Denkbar sind hier neben Cri[spi fil(ius) l(ibens) m(erito)] ferner die Lesungen Cri[spini f(ilius) l(ibens) $m$ (erito)] oder ähnlich. ${ }^{13}$ Zudem lässt sich eine fünfte Zeile nicht ausschließen. Die Lesung könnte in diesem Fall etwa Cri[spini fil(ius) / l(ibens) m(erito)] oder ähnlich gelautet haben. Dabei müsste man davon ausgehen, dass die Buchstaben LM offenbar zentriert in der Mitte der Zeile standen.

Die Matronae Arsacae sind durch eine seit langem verlorene nur handschriftlich bezeugte Inschrift aus Xanten/CUT bekannt. ${ }^{14}$ Zwar wurde der von TURCK und EWICH bezeugte Beiname Arsacis oftmals $\mathrm{zu}<M>$ arsacis korrigiert, da man von

\footnotetext{
${ }^{9}$ S. noch Galsterer 1983, 178-179. Nr.7. Abb.7 (Photo) mit der Lesung [.]ar[---]. Im Kommentar zur Inschrift erwägen B. und H. GALSTERER eine Ergänzung des Beinamens zu [M]ar[sacis] oder [P] ar[cis]. Vgl. auch ECK/KOßMANN 2009, 80 mit der Lesung [.]ar[---].

${ }_{10}$ Mit den Buchstaben AR beginnt nur noch der in Vettweiß-Müddersheim (Kreis Düren) belegte Matronenbeiname Arvagastae (CIL XIII 7855 = LEHNER 1918, 125. Nr.261). In der vorliegenden Inschrift scheint der Platz für Arvagastis jedoch nicht auszureichen.

${ }^{11}$ Grundsätzlich kann auch das seltenere Ma[tribus] nicht ausgeschlossen werden.

12 Zu Successus s. KaKoschKe 2008, 361. CN 2952. - Ein aus Successus gebildetes Pseudogentiliz Successius ist bisher nicht bezeugt, aber durchaus denkbar.

${ }^{13} \mathrm{Zu}$ Crispus bzw. Crispinus s. KAKOSCHKE 2007, 274-275. CN 961-962.

${ }^{14}$ CIL XIII 8630.
} 
einer Verbindung zu der germanischen gens der Marsaci ausging, ${ }^{15}$ doch besteht dazu offenbar keine Veranlassung. Eine weitere fragmentarische Inschrift aus Xanten/CUT beginnt mit M(atronis) Brittis Ma[rs?]acis. ${ }^{16}$ Lesung und Ergänzung der Inschrift bleiben jedoch äußerst unsicher. ${ }^{17}$ Somit handelt es sich bei dem Matronenbeinamen Marsacae offensichtlich um eine neuzeitliche Fiktion.

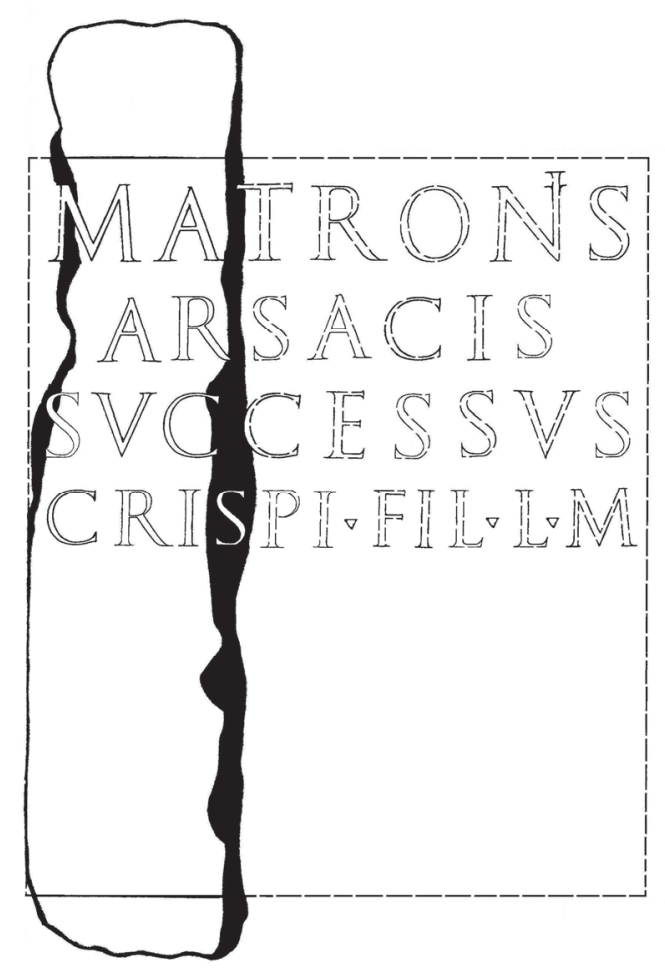

Abb.4: Leicht idealisierte Rekonstruktionszeichnung der fragmentarischen Weihinschrift IKöln ${ }^{2}$ 144. Nr.150 aus Köln/CCAA (Zeichnung A.K.)

\footnotetext{
${ }^{15}$ Die Korrektur schlug erstmals TH. BERGKS, WZ 1 (1882), 153. Anm.2 vor. Dem Vorschlag folgten M. SIEBouRG, WZ 6 (1887), 283, M. IhM, BJb 83 (1887), 18, 154-155. Nr.330, ders., RE II.1 (1895), 1267 (s.v. Arsaciae), SCHÖNFELD 1911, 30-31, GUTENBRUNNER 1936, 221. Nr.79-2, E. A. PHILIPPSON, The Germanic Review 19 (1944), 89. Aus der neueren Literatur s. u.a. SIMEK 1984, 252, R. HAENSCH, KJb 34 (2001), 127. Nr.175, ReUTER 2012, 114. Nr.69. Vgl. dagegen u.a. F. KAUfFMANN, Zeitschrift des Vereins für Volkskunde 2 (1892), 37, RieSE 1914, 326. Nr.3094, F. HeichelheIM, RE XIV.2 (1930), 2218 (s.v. Matres), REICHERT 1987-90, Teil 1, 494 („Emendation zu Marsacis fraglich“), SCHEID 2006, 336, DelamarRe 2007, 26. JufER/LuginBÜHL 2001, 23, 51 führen beide Lesevarianten auf.

${ }^{16}$ CIL XIII 8632 = RIESE 1914, 151. Nr.1367 = GUTENBRUNNER 1936, 221. Nr.79-1 = REUTER 2012, 139-140. Nr.106. S. auch JUFER/LUGINBÜHL 2001, 51.

17 In der EDCS Nr.11100726 lautet die Lesung M(atribus) Brittis M(atribus) A[rs]acis. Dieselbe Lesung geben Scheid 2006, 336 und Delamarre 2007, 26. S. ferner Spickermann 2005, 134 („,Möglicherweise gilt die Weihung ... neben den Matres Brittae auch den Matres Aresacae oder Arsacae ..."). Ältere Autoren boten noch die Lesung M BRITTIS MAX/ACIS [s. u.a. M. IHM, BJb 83 (1887), 19, 155. Nr.332]. Gemäß ZANGEMEISTER (Kommentar zu CIL XIII 8632) ist das X in MAXACIS jedoch unsicher. M.E. ist auf dem Stein M•BRITTIS•MAV/ACIS• zu lesen. Eine Ergänzung zu M(atribus) A[rs]acis bzw. Ma[rs]acis erscheint dagegen allein aus Platzgründen kaum möglich. S. auch schon M. IHM, BJb 83 (1887), 19.
} 
Für die fragmentarische Weihinschrift aus Köln/CCAA sei somit diese Lesung vorgeschlagen:

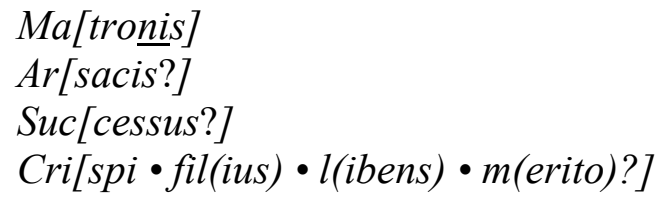

III.

Im Jahre 1874 entdeckte man in einem fränkischen Grab in einer Sandgrube am Schiefelsberg in Zülpich-Enzen (Kreis Euskirchen) einen Altar (Höhe $93 \mathrm{~cm}$ - Breite $53 \mathrm{~cm}$ - Tiefe $11 \mathrm{~cm}$ ) aus rötlichem Sandstein (Abb.5), der nur noch aus einer rechteckigen Platte besteht, da der Stein der Länge nach gespalten wurde. Für die Zweitverwendung als Umfassung eines Grabes hat man ferner den Sockel und das Gesims abgeschlagen. Auf den Schmalseiten sind noch Bäume zu erkennen. LEHNER gibt den Text des Steins in Umschrift wie folgt wieder: ${ }^{18}$
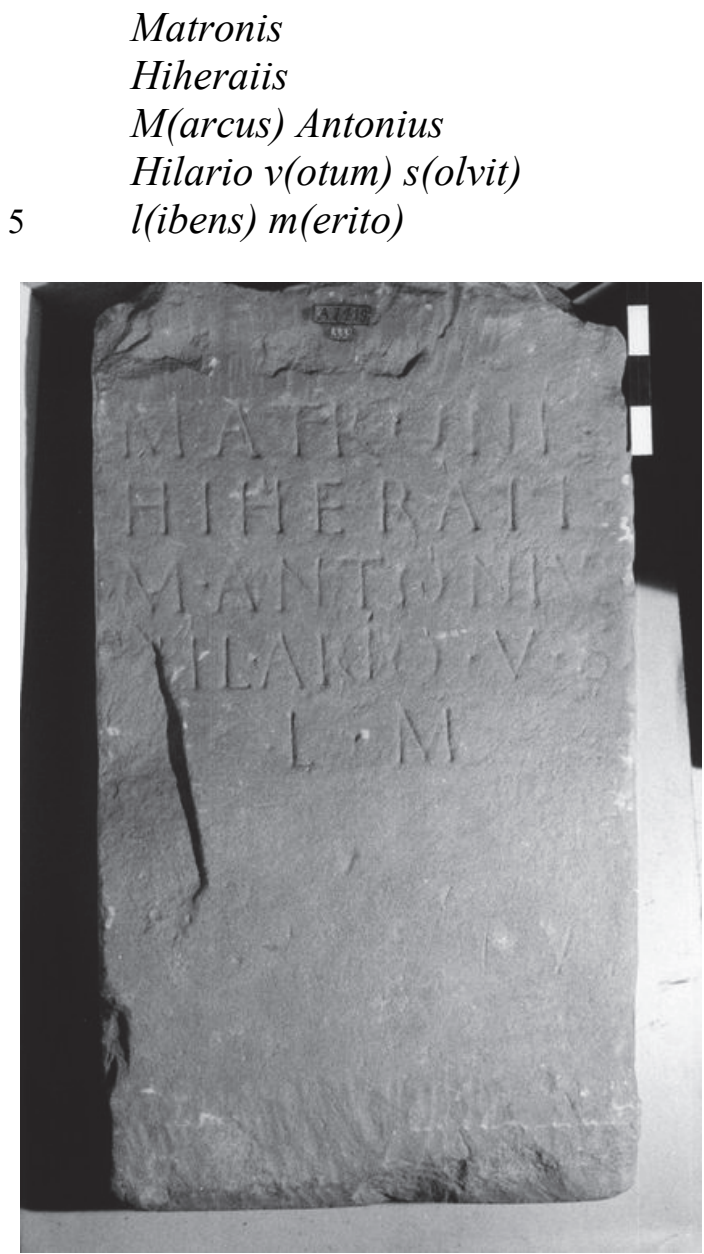

Abb.5: Weihinschrift aus Enzen: CIL XIII 7900

(C) CIL XIII/2-Projekt, Universität Trier)

\footnotetext{
${ }^{18}$ LEHNER 1918, 152. Nr.333. - Der Stein befindet sich heute im Rheinischen Landesmuseum in Bonn (Inv.-Nr.A1419).
} 
Die Inschrift bereitet bis auf die Lesung des Matronenbeinamens keine Probleme. In dem Wort bleibt der drittletzte Buchstabe unsicher. PoHL, der Ersteditor der Inschrift, schwankte zwischen den Buchstaben I, P und B und entschied sich schließlich für ein I, während der um Rat gefragte AUS'M WEERTH sich für ein abgeschliffenes R aussprach. ${ }^{19}$ Im Kommentar zur Inschrift heißt es im CIL aufgrund einer Autopsie ZANGEMEISTERs ,tertia a fine littera P potius fuit quam I“. Dagegen schreibt LEHNER: ,$\ldots$ ich halte den kleinen Bogen an dem vermeintlichen , $\mathrm{P}^{`}$ für eine zufällige Verletzung des Steins. “20 Spätere Autoren folgen mit der Lesung Hiherapis ZANGEMEISTER oder mit der Lesung Hiheraiis LEHNER bzw. POHL. ${ }^{21}$ Da bisher keine weiteren Belege für die Matronen vorliegen, ist die Lesung bis heute offen.

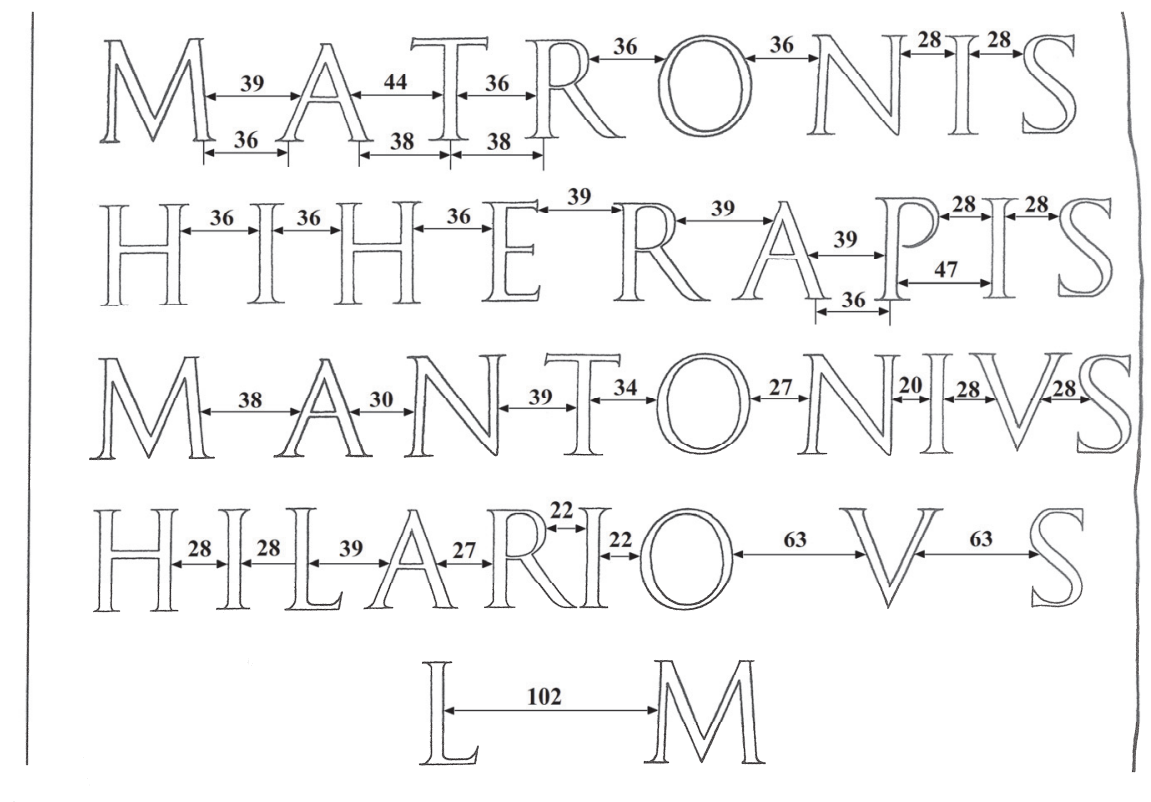

\begin{abstract}
Abb.6: Leicht idealisierte Rekonstruktionszeichnung der Weihinschrift CIL XIII 7900 aus Enzen mit Maßangaben in Millimeter (Zeichnung A.K.)
\end{abstract}

Augenscheinlich hat der antike Steinmetz die Inschrift recht sauber und durchdacht in den Stein gemeißelt. Eine Rekonstruktionszeichnung (Abb.6) demonstriert, dass die Laufweiten zwischen den einzelnen Buchstaben recht regelmäßig sind. Dies gilt umso mehr für die zweite Zeile. Insofern kann es sich bei dem drittletzten Buchstaben in Zeile 2 nur schwerlich um ein I handeln. Der Abstand zwischen dem vermeintlichen I und dem folgenden I wäre in diesem Fall mit $4,7 \mathrm{~cm}$ viel zu groß geraten. Einen vergleichbar großen Abstand zwischen zwei Buchstaben, vor allem zwischen zwei

\footnotetext{
${ }^{19}$ J. POHL, BJb 57 (1876), 83-85.

${ }^{20}$ LEHNER 1918, 152. Nr.333. S. auch LeHNER 1915, 197. Nr.A1419 (St.333).

${ }^{21}$ Für Hiherapis votieren u.a: SCHÖNFELD 1911, 135 [„Hiherap(i)ae“], REICHERT 1987-90, Teil 1, 427 („oder: hiheraiis“), JUFER/LUGINBÜHL 2001, 45, SCHEID 2006, 327 (mit falscher Fundortangabe), ECK/KoßMANN 2009, 76. - Für Hiheraiis votieren u.a.: M. IHM, BJb 83 (1887), 141. Nr.245 (im Kommentar heißt es jedoch: „Der drittletzte Buchstabe nicht ganz sicher; ich schwanke zwischen P und I“), F. KAUfFMAnN, Zeitschrift des Vereins für Volkskunde 2 (1892), 40 [,Hiheraiis (oder -apis?)“], J. B. KEUNE, RE VIII.2 (1913), 1592 (s.v. Hiheraiae) [„oder Hiherap(i)ae“, RIESE 1914, 330. Nr.3157, F. Heichelheim, RE XIV.2 (1930), 2227 (s.v. Matres), GutenbrunNER 1936, 217. Nr.67 (mit einem „?“), E. A. PhilipPson, The Germanic Review 19 (1944), 94. Anm.38, SimeK 1984, 179, 257, VENNEMANN 1993, 391-392, BEYER 1999/200, 183 (Hiheraihae!), KAKOSCHKE 2007, 407. CN 1520-1 („Hiheraiae?“), GARMAN 2008, 123. Nr.168, BILLER 2010, 120, EDCS Nr.11100125 (mit einem „,!“). WEISGERBER 1968, 27. Nr.125 liest „Hihera?is“.
} 
Buchstaben mit abschließenden senkrechten Hasten, wie N und I, H und I, H und $\mathrm{E}$ oder I und L, weist die Inschrift (sieht man von der letzten Zeile ab) an keiner anderen Stelle auf. Liest man jedoch mit ZANGEMEISTER statt eines I ein P, gestaltet sich die Laufweite in Zeile 2 absolut harmonisch. Die Lesung Hiheraiis sollte somit verworfen werden. Höchstwahrscheinlich führten die aus Enzen bekannten Matronen den Beinamen Hiherapae oder Hiherapiae.

IV.

Im Jahre 1937 zeigte WoELCKE mit einem kurzen Satz den Fund eines Bronzetäfelchens in Frankfurt-Praunheim an. Den Text des Täfelchens, das damals Eingang in die Sammlung des Stadtgeschichtlichen Museums in Frankfurt fand, gab WoELCKE zutreffend wie folgt wieder: ${ }^{22}$

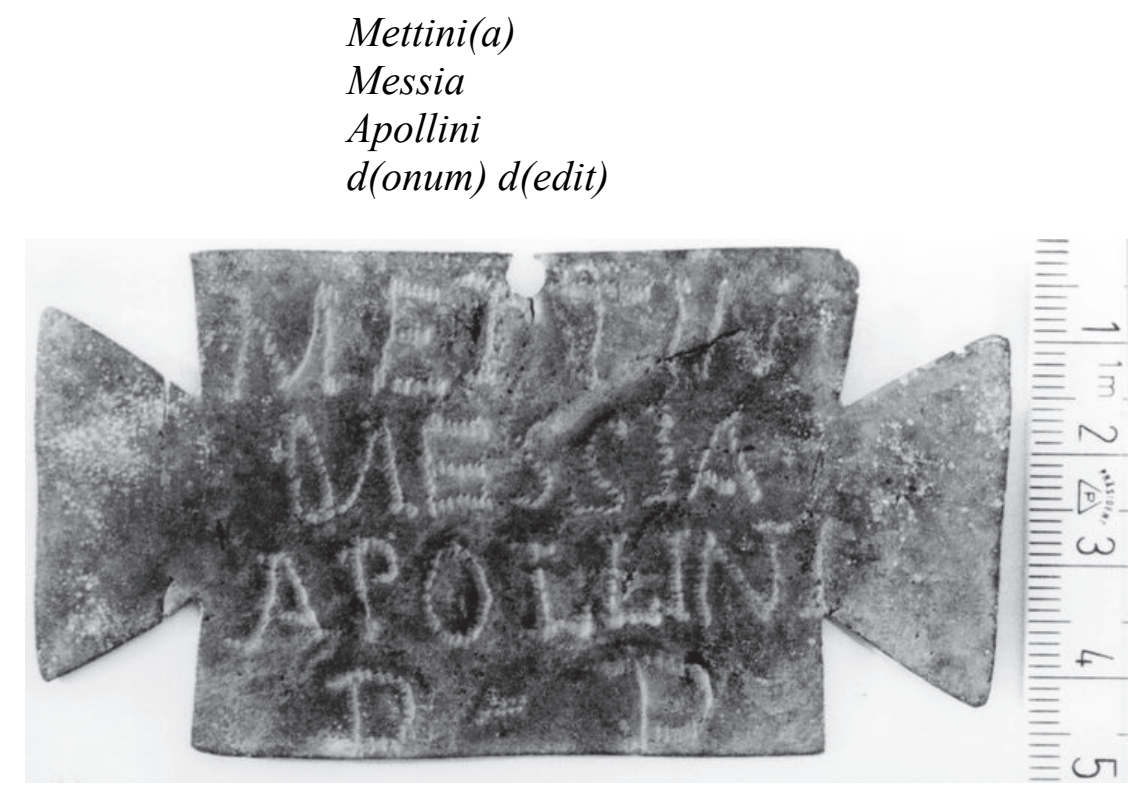

Abb.7: Weihinschrift aus Frankfurt-Heddernheim/Nida: K. WoELCKE, Germania 21 (1937), 131 (C) CIL XIII/2-Projekt, Universität Trier)

Da die Inschrift nicht in den Nachträgen zum CIL oder in der AE aufgenommen wurde, seien an dieser Stelle einige Bemerkungen zu dem Fund nachgetragen: Das Bronzetäfelchen (Höhe 4,7 cm - Breite $9 \mathrm{~cm}$ ) in Form einer Tabula ansata mit eingepunzter Inschrift (Abb.7) lässt sich laut SPICKERMANN nur grob der Zeit zwischen 100 und $250 \mathrm{n}$. Chr. zuordnen. ${ }^{23}$ Heute befindet sich das Stück im Archäologischen Museum in Frankfurt [Inv.-Nr.a12568 (Zugangsnr.1936/11)]. Das Stadtgeschichtliche Museum Frankfurt kaufte das Täfelchen am 16.12.1936 vom Hauptwachtmeister Merz (wohnhaft „Am Ebelfeld 224“ in Frankfurt-Praunheim). Entgegen den Angaben WOELCKEs verzeichnet das Hauptinventar des Archäologischen Museum als Fundort „Heddernheim, Parzelle 20/655“. Dieses Grundstück liegt im Westen von Heddernheim/Nida, unweit des nordöstlichen Stadttores. Bis auf einige Töpferöfen sind aus diesem Bereich keine größeren Baubefunde bekannt. Das Fixierungsloch im oberen Bereich des Bronzetäfelchens zeigt, dass das Objekt ursprünglich wohl an einem

\footnotetext{
${ }^{22}$ K. Woelcke, Germania 21 (1937), 131.

${ }^{23}$ SPICKERMANN 1994, 248. Nr.7.
} 
Weihegeschenk befestigt war. Möglicherweise darf man als Aufstellungsort des Weihegeschenks ein örtliches Heiligtum vermuten. ${ }^{24}$

Das Gentiliz der Dedikantin, das von WOELCKE wohl korrekt zu Mettini(a) aufgelöst wurde, ist nicht weiter bekannt. SPICKERMANN erblickt in Mettinius nicht zu unrecht „eine veränderte Schreibweise“ des italischen Gentilnomens Metinius. ${ }^{25}$ Für das seltene Metinius ist ein Beleg aus Dalmatien bezeugt. ${ }^{26}$ Zudem kann auf einen Prätorianer aus Rom namens Metinius Aper verwiesen werden. ${ }^{27}$ Es ist jedoch nicht auszuschließen, dass das aus Heddernheim/Nida bekannte Mettinia vom keltischen Cognomen Mettus abgeleitet wurde, für das sich ein Beleg in Trier/Augusta Treverorum findet. ${ }^{28}$ In diesem Fall dürfte man in der Dedikantin zweifellos eine Einheimische aus dem gallisch-germanischen Raum erblicken. Hierfür mag auch das Cognomen Messia sprechen, da der Name, bei dem es sich eigentlich um ein italisches Gentiliz handelt, vor allem in keltischen Gebieten verbreitet war. ${ }^{29}$

V.

Im Jahre 1910 entdeckte man in Zülpich-Merzenich (Kreis Euskirchen) eine fragmentarische Weihinschrift für die Matronae Cuchenehae. Die Inschrift steht auf einer Platte aus Sandstein (Höhe $66 \mathrm{~cm}$ - Breite $48 \mathrm{~cm}$ - Tiefe $11 \mathrm{~cm}$ ), die sich aus drei Bruchstücken zusammensetzt (Abb.8). LEHNER gibt die vierzeilige Inschrift wie folgt wieder: ${ }^{30}$

\section{Matronis \\ Chuhenehi[s] \\ M(arcus) Me[.]iniu[s] \\ Tert i] us l(ibens) m(erito)}

Bis auf das Gentiliz des Dedikanten bereitet die Lesung der Inschrift keine Schwierigkeiten. GUTENBRUNNER erblickt in dem Gentilnomen eine Ableitung vom keltischen (oder griechischen) Cognomen Melus und ergänzt den Namen zu dem nicht weiter bezeugten Me[l]inius. ${ }^{31}$ Eine Rekonstruktionszeichnung der Inschrift (Abb.6) zeigt jedoch, dass die Lücke in Zeile 3 für ein L etwas zu groß ist.

\footnotetext{
${ }^{24}$ Aus Heddernheim/Nida ist ein weiteres fragmentarisches Bronzetäfelchen für Apollo bekannt: AE $1977,591=4$. N. 512 . Nr.108.

25 SpickermanN 1994, 251. Anm.143. Zum Namen s. ferner Schulze 1904, 188, KaKOSChKe 2006, 273. GN 799.

${ }^{26}$ EDCS Nr.67300133 (Stari Grad/Argyruntum).

${ }^{27}$ CIL VI $2438=$ EDR Nr.103663.

${ }^{28}$ AE 1964, $149=4$. N. 452. Nr.1. - Ferner führen HARTLEY/DiCKINSON 2010, 97-98 einen Töpfer namens Mettius (oder Mettus?) für Lezoux (dép. Puy-de-Dome) im Gebiet der Arverni und mit Fragezeichen einen Töpfer namens Mettus für Rheinzabern/Tabernae. Zu Mettus s. auch KAKOSCHKE 2010, 415. CN 948.

${ }^{29} \mathrm{Zu}$ Messius als Cognomen s. KaKoschKe 2008, 115. CN 2034. Zu Messius als Gentilnomen s. KAKOSCHKE 2006, 271-272. GN 791.

${ }^{30}$ LEHNER 1915, 200, LEHNER 1918, 147-148. Nr.325. Mit identischer Lesung (durch FINKE) fand die Inschrift Aufnahme in das CIL (XIII 12009) und in EDCS Nr.12800030. Vgl. ferner WEISGERBER 1968, 47. Nr.856, GARMAN 2008, 121. Nr.158, BILler 2010, 117. Anm.596. - Die Inschrift befindet sich heute im Rheinischen Landesmuseum in Bonn (Inv.-Nr.22346).

${ }^{31}$ Gutenbrunner 1936, 217. Nr.69-2. Zu Melus s. KaKoschKe 2008, 110. Nr.2012.
}

http://www.fera-journal.eu 


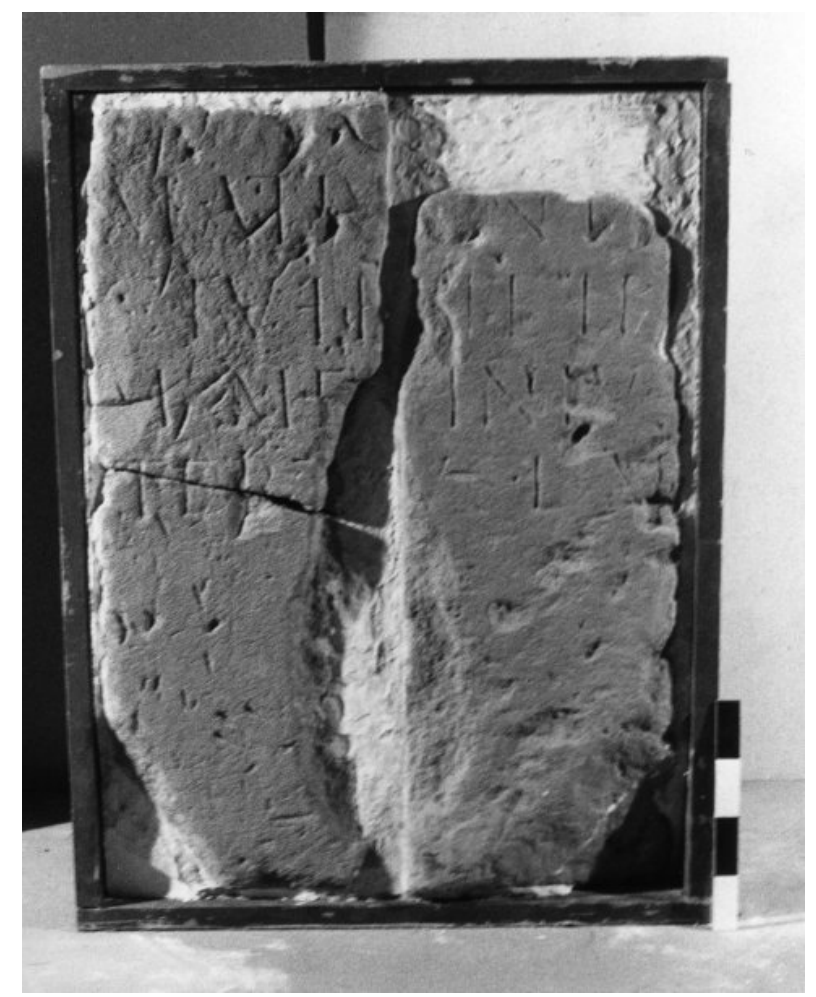

Abb.8: Fragmentarische Weihinschrift aus Merzenich: CIL XIII 12009 (C) CIL XIII/2-Projekt, Universität Trier)

Geht man davon aus, dass in Me[.]inius ein Buchstabe fehlt, kommt für eine Ergänzung aus dem Repertoire der bisher bekannten Gentilnomina nur Metinius in Frage, ${ }^{32}$ zumal $\mathrm{T}$ im Gegensatz zu anderen möglichen Buchstaben in die vorhandene Lücke problemlos eingepasst werden kann (Abb.9). Das Gentiliz ist, wie oben bereits dargelegt, relativ selten. Entweder führte der Dedikant aus Zülpich-Merzenich daher einen seltenen italischen Namen oder einen einheimischen -inius-Namen, der sich vom Cognomen Mettus ableitet. Die Schreibung des Namens mit einem T muss dabei nicht gegen eine Gleichsetzung des Namens mit der aus Heddernheim/Nida bezeugten Variante des Namens mit zwei $\mathrm{T}$ sprechen, da entsprechende Wechsel von geminierten und nicht geminierten Buchstaben innerhalb eines Namens nicht nur im Namenmaterial der zwei germanischen Provinzen oftmals bezeugt sind..$^{33}$

\footnotetext{
${ }^{32}$ S. SOLIN/SALOMIES 1994, 116-119.

${ }^{33}$ Auf folgende Fälle aus den zwei germanischen Provinzen sei hier beispielhaft verwiesen: CIL XIII *1328 [Nijmegen/Ulpia Noviomagus; C. Metius(!) Martialis], 8275 (Köln/CCAA; C. Vetienius(!) C. f. Pup. Urbiqus], 8556 [Neus/Novaesium; M. Metius(!) [..] f. Macellus], 11984 (Nettersheim; M. Aurelius Agripinus(!), 11989 [Nettersheim; Pettronius(!) Patroclus]. Zu weiteren Belegen s. KAKOsCHKE 2014, 46. Anm.195-196.
} 


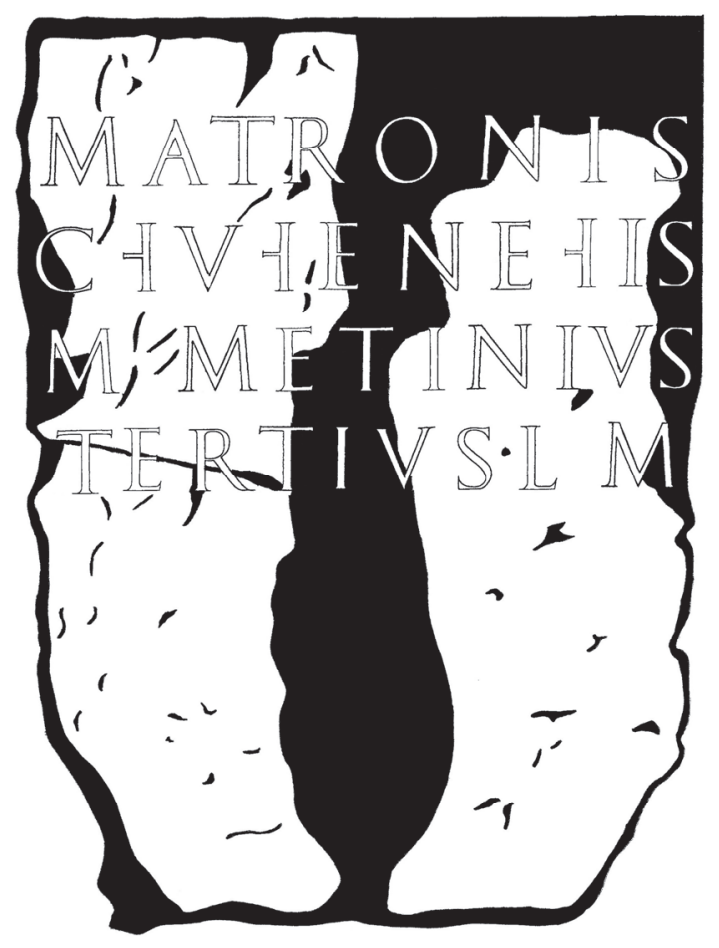

Abb.9: Leicht idealisierte Rekonstruktionszeichnung der fragmentarischen Weihinschrift CIL XIII 12009 aus Merzenich (Zeichnung A.K.)

VI.

Im Bereich des Kastells Krefeld-Gellep/Gelduba fand sich im Jahre 1970 das Fragment einer schmalen Bronzetafel (Höhe 7,3 cm - Breite 10,5 cm - Tiefe 0,4 cm), das nur im oberen und unteren Bereich vollständig erhalten ist (Abb.10). ROTHENHÖFER, der Ersteditor der Inschrift, gibt den Text des Fragments in Majuskelschrift zu Recht wie folgt wieder: ${ }^{34}$

$$
\begin{aligned}
& \text { DEAE AH[---] } \\
& \text { [---]ALIS FRO[---] }
\end{aligned}
$$

Da sich in Chesterholm/Vindolanda eine von der [co]h(ors) I Tungr[orum (milliaria)] dedizierte Weihinschrift für die Ahvardua dea fand, ${ }^{35}$ schlägt ROTHENHÖFER (mit leichten Bedenken) für die erste Zeile der Bronzetafel die Lesung Deae Ah[varduae] vor. ${ }^{36}$ Genau genommen müsste es natürlich [---] Deae Ah [varduae?] heißen. ${ }^{37}$

\footnotetext{
${ }^{34}$ ROTHENHÖFER 2014, 28. - Die noch zu erkennende rechte Vertikale des A am Beginn der zweiten Zeile passt im Winkelmaß nicht ganz zu den entsprechenden Hasten der noch vorhandenen Buchstaben A in der ersten Zeile. Das A der zweiten Zeile weist im unteren Bereich einen größeren Abstand zwischen den Vertikalen auf. Zudem ist der Abstand zu dem folgenden L aufgrund einer auslaufenden Serife relativ groß geraten. Eine alternative Lesung bietet sich für den Beginn der zweiten Zeile aber nicht an, da es sich hier eindeutig um ein A handelt und eine AL-Ligatur aufgrund der Serifenform nicht zu entziffern ist. - Die Bronzetafel befindet sich heute im Museum Burg Linn in Krefeld (Inv.Nr.70/2001).

35 A. R. BIRlEy u.a., ZPE 186 (2013), 287-288. Abb.1-2 (Photo + Zeichnung), R. S. O. TomLIN, Britannia 44 (2013), 384-385. Nr.5. Abb.5 (Photo).

${ }^{36}$ ROTHENHÖFER 2014, 28-29.

37 Wenn der Göttername vorangestellt wurde, wie in der von ROTHENHÖFER zitierten Inschrift aus Chesterholm/Vindolanda, ist in der ersten Zeile grundsätzlich auch eine Lesung wie z.B. [Fortunae]
} 


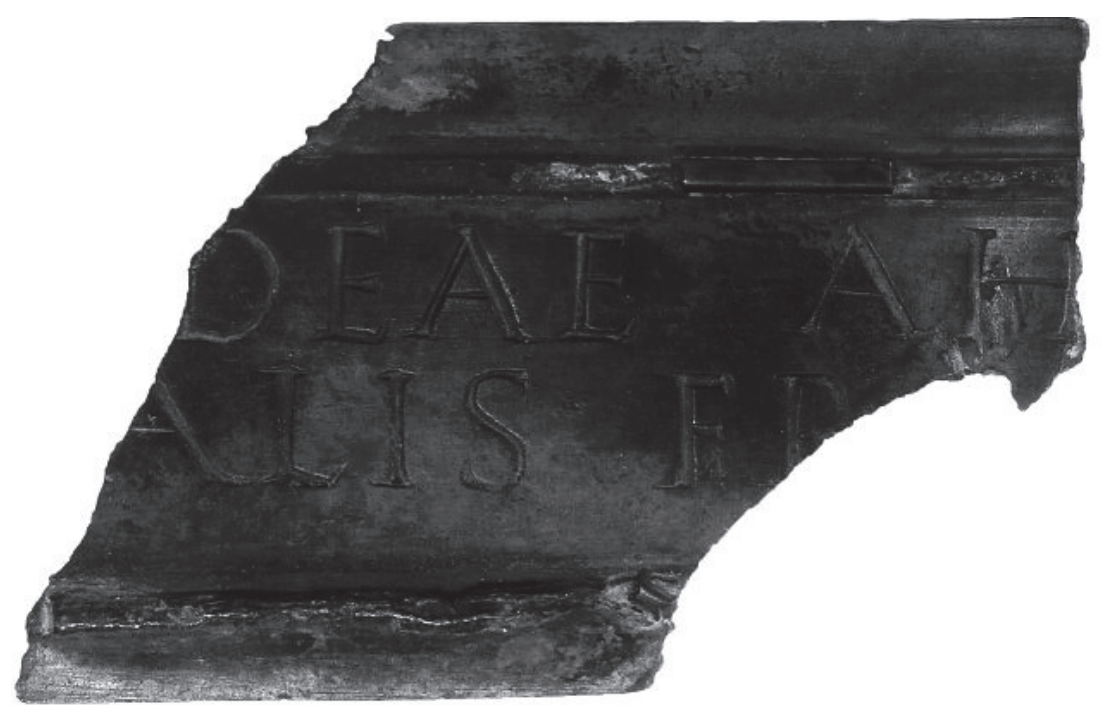

Abb.10: Fragmentarische Weihinschrift aus Krefeld-Gellep/Gelduba: ROTHENHÖFER 2014, 29. Abb.1 (C) EDCS Nr.68800001/Rothenhöfer)

Für die Ergänzung der zweiten Zeile wägt ROTHENHÖFER ausführlich mehrere Möglichkeiten ab. Dabei schließt er ein seltenes auf -alis endendes Gentilnomen und ein mit Fro- beginnendes Cognomen ebenso aus wie die Nennung einer Person mit zwei Cognomina. Ferner verwirft der Autor die mögliche Nennung mehrere Dedikanten, da zwischen [---]alis und Fro[---] ein verbindendes et fehlt. Die Überlegungen führen ROTHENHÖFER schließlich zu der gewagten Lesung „[equites vel sim. ex] alis Fro[ntoniana et --- v(otum) s(olverunt) vel sim.]". Die Nennung der ala Frontoniana scheint dem Autor dabei wahrscheinlich, da die Reitereinheit in claudisch-neronischer Zeit im nahen Asberg/Asciburgium stationiert war. ${ }^{38}$ Gleichzeitig gesteht er jedoch ein, dass die Tafel ,offensichtlich bereits in der Antike intentionell zerkleinert (wurde), um das Metall der Wiederverwendung zuzuführen." Und da Altmetall über weite Strecken transportiert wurde, bleibt der Aufstellungsort der Bronzetafel - wie ROTHENHÖFER eingesteht - völlig unsicher. ${ }^{39}$

Gegen die Lesung RoTHENHÖFERs spricht aber vor allem die Tatsache, dass ein dem (einheimischen) Götternamen vorangestelltes dea oder deus frühestens in hadrianischer Zeit auftritt, wobei die allermeisten Belege aus der Zeit zwischen 170 und $230 \mathrm{n}$. Chr. stammen. ${ }^{40}$ ROTHENHÖFER gesteht diese Schwäche seiner Lesung auch ein, möchte aber aufgrund seiner Ergänzungen den chronologischen Rahmen für ein vorangestelltes dea oder deus ,deutlich in das erste Jahrhundert der Kaiserzeit (ausweiten)“. ${ }^{41}$ Die Basis für eine entsprechende Änderung der Chronologie ist dabei aber sicher zu schmal, zumal sich für die zweite Zeile der Tafel auch eine recht un-

deae Ah[ucco Leubasni filius] nicht auszuschließen. Der Einwand zeigt nur die grundsätzlichen Schwierigkeiten bei der Ergänzung einer nur aus wenigen Buchstaben bestehenden Inschrift auf. - Zudem sei auf die wenigen Belege für ein nachgestelltes deae/deo in den Inschriften aus den zwei germanischen Provinzen verwiesen: CIL XIII 6262 (Alzey/Altiaia; Fortunae deae), 7504 (Bingen/Bingium; Sulevis dea[bus]), 2. N. 76-77. Nr.84 (Alzey/Altiaia; Apollini deo Demionco), 4. N. 486. Nr.53 [Obernburg am Main; S(oli) d(eo) i(nvicto) M(ithrae)], AE 1976, $501=1987,782$ (Mainz/Mogontiacum; Silvano deo), 1991, 1256 (Avenches/Aventicum; Livillae deae). Zum Phänomen s. M.-TH. RAEPSAET-CHARLIER, ZPE 61 (1985), 204-208, RAEPSAET-CHARLIER 1993, 12, 66.

${ }^{38}$ ROTHENHÖFER 2014, 29-30. Zur Einheit s. ALFÖLDY 1968, 38-40, SPAUL 1994, 117-119.

${ }^{39}$ ROTHENHÖFER 2014, 27-28.

${ }^{40}$ RAEPSAET-CHARLIER 1993, 12-15. S. ferner u.a SPICKERMANN 1994, 13.

${ }^{41}$ ROTHENHÖFER 2014, 30-31. 
spektakuläre Lesung anbietet. So könnte [---]alis Fro[---] zu einem einheimischen Namen, wie z.B. [Vit]alis Fro[ntonis fil(ius)] oder [Iulius Nat]alis Fro[ntini fil(ius)] ergänzt werden - eine einfache und daher bestimmt überzeugendere Möglichkeit, die von ROTHENHÖFER offensichtlich übersehen wurde. ${ }^{42}$

\section{VII.}

Aus Köln/CCAA stammt ein wohl im vierten Jahrhundert für eine Sekundärverwendung in fünf senkrechte Platten zersägter marmorähnlicher Kalksteinblock (Höhe $70,5 \mathrm{~cm}$ - Breite $66 \mathrm{~cm}$ - Tiefe $75 \mathrm{~cm}$ ) mit Inschrift (Abb.11). Die heute verlorenen Platten fanden sich im Jahre 1925 in einer Grabkammer im Kreuzgang von St. Severin. ${ }^{43}$ B. und H. GALSTERER datieren den Block PÄFFGEN folgend in das zweite oder dritte Jahrhundert n. Chr., ${ }^{44}$ während GREGAREK sich aufgrund von stilistischen Kriterien für eine Datierung in das erste Jahrhundert n. Chr. ausspricht. ${ }^{45}$ Der von einem Eichenkranz gerahmten Text des unvollständigen Blocks wird von B. und H. GALSTERER in Umschrift wie folgt wiedergegeben: ${ }^{46}$

$\begin{array}{ll} & \text { Primio } \\ & \text { Cellissi fil(ius) } \\ & \text { curia(lis) gru(e)s duas } \\ & \text { Mercurio } \\ 5 \quad & v(\text { otum) s(olvit) l(ibens) m(erito) }\end{array}$

Die Lesung der Inschrift bereitet bis auf Zeile 3 keine Probleme. FREMERSDORF, der Ersteditor der Inschrift, interpretierte CVRIA zunächst als Herkunftsangabe (,aus Chur" $),{ }^{47}$ verwarf den Gedanken aber wieder und sah das „Rätsel“ des Wortes für „,noch zu lösen“ an. Dagegen erklärte FREMERSDORF mit Bestimmtheit, dass das folgende GRVSDVAS nur als ,gru(e)s duas $=$ zwei Kraniche“ zu verstehen ist. ${ }^{48}$ In diesem Sinne interpretierte auch FINKE die Buchstabenfolge, der erstmals von zwei „wohl aus Bronze gegossenen Kraniche(n) als Votivgabe“ ausging. Zudem löste FINKE CVRIA zu curia(lis) auf und sah in dem Dedikanten ein Mitglied des ordo decurionum oder den Innhaber eines kommunalen oder priesterlichen Amtes. ${ }^{49}$

\footnotetext{
${ }^{42}$ Vgl. auch noch EDCS Nr. 68800001. In der Datenbank wurde die vorgeschlagene Lesung RoTHENHÖFERs nicht übernommen.

${ }^{43}$ Der Block ist seit dem Zweiten Weltkrieg verloren. Das Römisch-Germanische Museum in Köln führt den Stein noch mit der Inv.-Nr.25,811. Zur Fundsituation s. PÄFFGEN 1992, Bd.2, 81-82.

${ }^{44} \mathrm{IKöln}^{2}$ 160, PÄFFGEN 1992, Bd.2, 83.

${ }^{45}$ H. GReGAREK, KJb 37 (2004), 58. S. auch SPICKERMANN 2008, 129.

${ }^{46}$ IKöln $^{2}$ 159-160. Nr.172. Die fehlerhafte Wiedergabe von Cellissi mit nur einem L wurde hier korrigiert. Derselbe Fehler findet sich u.a. auch bei SCHOPPA 1959, 20, GALSTERER 1975, 34-35. Nr.118. Taf.25 (Photo), PÄFfGEN 1992, Bd.2, 82, H. GREGAREK, KJb 37 (2004), 56, SPICKERMANN 2008, 122. Nr.21, 129. Anm.215, DONDIN-PAYRE 2012, 88-89. Nr.12, A. SCHÄFER, KJb 45 (2012), 537. - Die Zusammengehörigkeit der Platten ist nicht zuletzt aufgrund der Fundsituation gesichert. Offenbar lässt sich die fehlende Passgenauigkeit zwischen den Buchstaben G und C in Zeile 3 und 4 dadurch erklären, dass durch den Vorgang des Zersägens Material verloren ging. Auf dem hier vorgelegten Photo stehen die Platten dadurch etwas zu nah beieinander.

${ }^{47}$ F. FremersDORF, BJb 130 (1925), 281. S. auch noch SCHOPPA 1959, 20.

${ }^{48}$ F. FREMERSDORF, Germania 10 (1926), 116-117.

49 1. N. 96. Nr.290.
} 


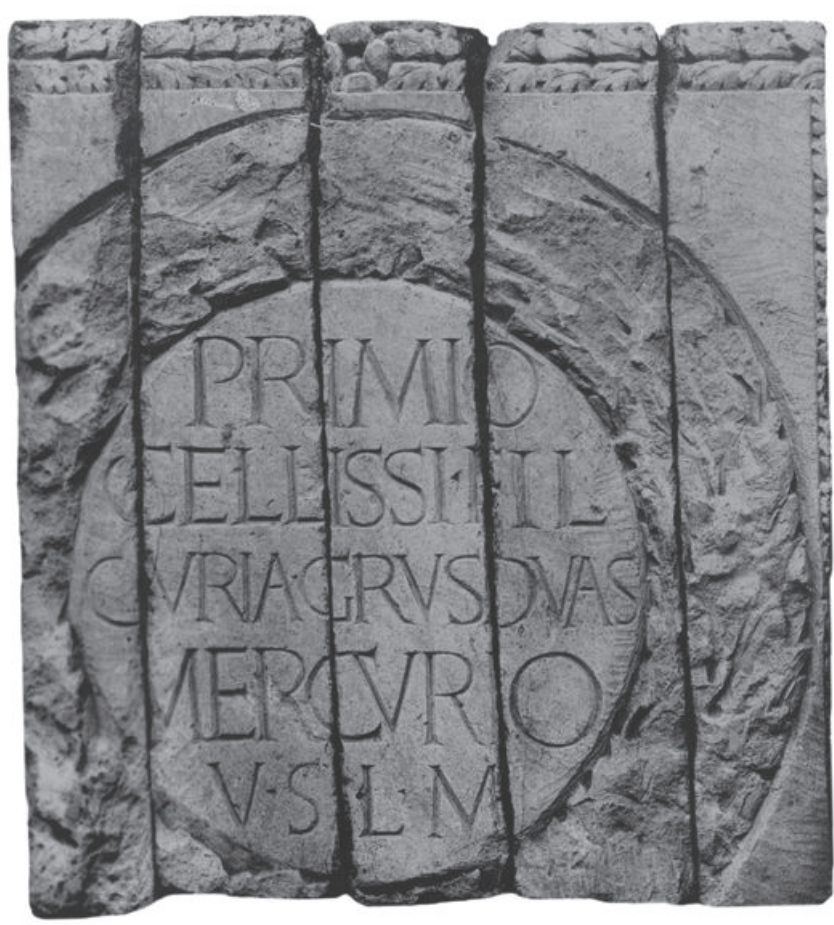

Abb.11: Weihinschrift aus Köln/CCAA: 1. N. 97 . Nr.290 (C) Römisch-Germanisches Museum, Köln)

Diesen alten Auffassungen entsprechend begreifen auch noch B. und H. GALSTERER die Inschrift. Allerdings weisen Sie zu Recht darauf hin, dass der Begriff curialis für ein Mitglied der städtischen curia erst in nachkonstantinischer Zeit aufkommt. Ferner gehen Sie davon aus, dass der Dedikant, Primio Cellissi fil., kein decurio der CCAA gewesen sein kann, da er wie es seine Namensform andeutet offenbar nicht im Besitz des römischen Bürgerrechts war. RÜGER folgend sehen B. und H. GALSTERER in Primio Cellissi fil. vielmehr ein Mitglied einer gallisch-germanischen Kurie, wie sie aus dem Umkreis von Matronenheiligtümern bezeugt ist. ${ }^{50}$

B. und H. GALSTERER teilen ferner die Auffassung von GREGAREK, der zufolge die in der Inschrift genannten Kraniche auf dem Votivsockel in Form von Bronzestatuen aufgestellt waren. ${ }^{51}$ Wie bereits FREMERSDORF, FINKE und RÜGER betonen B. und $\mathrm{H}$. GALSTERER dabei ausdrücklich die engen Verbindungen zwischen dem keltischen Merkur, den rheinischen Matronen und dem Kranich. ${ }^{52}$

Diesen Vorstellungen ist folgendes entgegenzustellen: Die Lesung von CVRIA im Sinne von curia(lis) ist problematisch, da keine weiteren Belege für die Abkürzung curia(lis) oder decuria(lis) vorliegen. Daher sollte man CVRIA vielleicht eher als curia (= Kurie) lesen. Diese Lesung bietet neuerdings auch DONDIN-PAYRE, die jedoch bei der Lesung gru(e)s duas bleibt. ${ }^{53}$ Bereits FrEMERSDORF hatte jedoch darauf verwiesen, dass in Verbindung mit dem keltischen Merkur stets drei - und

\footnotetext{
${ }^{50}$ IKöln $^{2}$ 159-160. Nr.172 unter Verweis auf RÜGER 1987, 26-27. - Zu den in Niedergermanien und der Gallia Belgica bezeugten Kurien s. grundsätzlich RÜGER 1972, BILLER 2010, 289-294, DONDIN-PAYRE 2012.

${ }^{51}$ H. GREGAREK, KJb 37 (2004), 56-58. Abb.22 (Rekonstruktionszeichnung des Votivsockels mit zwei bronzenen Kranichen). Die Zeichnung findet sich auch unter IKöln² 159-160. Nr.172.

52 IKöln ${ }^{2}$ 159-160. Nr.172 unter Verweis auf RÜGER 1987, 26-27. S. ferner F. FREMERSDORF, Germania 10 (1926), 116-117, 1. N. 96. Nr.290.

${ }^{53}$ DONDIN-PAYRE 2012, 88-89. Nr.14.
} 
nicht zwei - Kraniche als Attribute bildlich genannt werden. ${ }^{54}$ Der Lesung gru(e)s duas und der daraus resultierenden ,schwer verständlichen Weihung von zwei Kranichen an Merkur" ${ }^{\text {"55 }}$, für die es in der gesamten lateinischen kaiserzeitlichen Epigraphik keine Parallele gibt, ${ }^{56}$ ist aber vor allem der epigraphische Befund entgegenzusetzen: Auf dem Stein finden sich sorfälltig eingeschlagene Worttrenner in Form eines Dreiecks zwischen CELLISSI und FIL (Zeile 2), zwischen CVRIA und GRVSDVAS (Zeile 3) und zwischen den Buchstaben der Schlussformel VSLM (Zeile 4). Zwischen GRVS und DVAS fehlt jedoch ein Worttrenner. Der Umstand wurde bereits von FREMERSDORF ausdrücklich bemerkt. ${ }^{57}$ Demzufolge ist GRVSDVAS wohl als ein Wort anzusehen. Diese Vermutung wird durch den Fund eines Steins aus AltInden (Kreis Düren) bestätigt, der den (Matronae) Grusduahenae geweiht war. Laut NeDOMA, der den Neufund erstmals korrekt las, ${ }^{58}$ zählt der Name zur Gruppe der topischen Matronenbeinamen. NedomA zufolge hat der Wortstamm Grusdua- die Bedeutung „kiesiger Ort, Schotterplatz, Geröllgelände“. 59 Somit scheint sich für das durch den Kölner Stein bezeugte GRVSDVAS eine alternative Lesung anzubieten. Zudem bezieht sich GVRSDVAS offenbar auf CVRIA. Insofern lösen sich die Zweifel von DONDIN-PAYRE auf, die sich noch darüber erstaunt zeigte, dass in der Kölner Inschrift eine curia ohne Namen genannt wird. ${ }^{60}$ Die namentliche Nennung einer curia passt auch zu den drei weiteren bekannten Tituli aus dem Bereich der niederrheinischen Matroneninschriften, die eine curia nennen, da zumindest zwei dieser Inschriften ebenfalls dem Merkur geweiht waren. ${ }^{61}$

Die Angaben in der dritten Zeile sind vielleicht als Herkunftsangabe, im Sinne von (ex) curia Grusduas zu verstehen. Denkbar ist ferner die Deutung von curia Grusduas als Nominativ. Dann müsste man davon ausgehen, dass die Weihung von Primio Cellissi fil. und der curia vorgenommen wurde. Für diese Variante spricht vielleicht, dass Grusduas noch am ehesten als Nominativ Singular anzusehen ist. Die Form Grusduas bereitet grundsätzliche Schwierigkeiten und ist sicher der Schwachpunkt der hier vorgetragenen Lesung. In Analogie zur bekannten curia Amratnina ${ }^{62}$ hätte man statt Grusduas ein ähnlich gebildetes Adjektiv, wie *Grusduanina, oder in Analogie zur curia Etratium ${ }^{63}$ einen Gruppennamen im Genitiv Plural, wie etwa *Grusduat(i)um, erwartet. Dabei muss jedoch betont werden, dass bisher nur zwei Kuriennamen epigraphisch bezeugt sind und weitere Namen nur theoretisch er-

54 F. Fremersdorf, Germania 10 (1926), 116 unter Verweis auf die Steine CIL XIII 3026 (Paris/Lutetia) und CIL XIII 3656 (Trier/Augusta Treverorum) sowie auf den Grabstein eines Remers (CIL XIII 8104, Bonn/Bonna), der ebenfalls drei Kraniche zeigt. Zur Verbindung von Merkur mit drei Kranichen s. auch A. Ross, Études celtique 9 (1961), 405-438.

55 SCHOPPA 1959, 21.

${ }^{56}$ S. auch SPICKERMANN 2008, 129.

${ }^{57}$ F. FREMERSDORF, Germania 10 (1926), 116. - S. auch noch FINKE (1. N. 96. Nr.290), der bei seiner Wiedergabe der Inschrift in Majuskelschrift zwischen GRVS und DVAS eine Lücke lässt, die auf dem Stein nicht existiert. Eine entsprechende Wiedergabe bietet AE 1926, 18. - B. und H. GALSTERER (IKöln ${ }^{2} 159-160$. Nr.172) geben die Inschrift ohne Worttrenner wieder, obwohl in den IKöln ${ }^{2}$ bei vielen Umschriften auch die Worttrenner berücksichtigt werden.

${ }^{58}$ B. BEYER / B. PÄFFGEN, Archäologie im Rheinland 2006, 132-133 (= AE 2006, 865) lasen den Anfang der Inschrift noch als ,an den Kranich der duahenischen Matronen (GRVS DVAHENIS)“. S. dazu bereits die kritischen Anmerkungen von BILLER 2010, 262.

${ }^{59}$ NEDOMA 2014, 441-447.

${ }^{60}$ DONDIN-PAYRE 2012, 88-89. Nr.14. S. auch SPICKERMANN 2008, 129, der jedoch davon ausgeht, dass der auffälligerweise fehlende Name auf ein „Organ der ubischen civitas“ deutet.

${ }^{61}$ CIL XIII 7859 (Weisweiler), 4. N. 529-530. Nr.147 = AE 1972, 357 (Holzweiler). In der Inschrift 4. N. 529. Nr.145 = AE 1972, 356 (Laurenzberg) wird MERCVRIO am Anfang ergänzt.

${ }^{62}$ CIL XIII 7859 (Weisweiler), 4. N. 529. Nr.145 = AE 1972, 356 (Laurenzberg).

63 4. N. 529-530. Nr.147 = AE 1972, 357 (Holzweiler). 
schlossen wurden. ${ }^{64}$ Die Form Grusduas lässt sich hier auf jeden Fall nicht abschließend bestimmen. Handelt es sich vielleicht um eine Abkürzung oder liegt eine einheimische Flexion vor? ${ }^{65}$

Für die Lesung der dritten Zeile bietet sich noch eine weitere Möglichkeit an: Die Namen der bisher bekannten Kurien und die Herleitung dieser Namen aus Matronen- bzw. Gruppennamen führen RÜGER zu der interessanten Überlegung, dass in Niedergermanien möglicherweise auch Gentil- und Cognomina existierten, die aus Matronenbeinamen gebildet wurden. ${ }^{66}$ Dabei kann RÜGER, der ausdrücklich von einer theoretischen Möglichkeit spricht, jedoch nur auf ein relativ überzeugendes Beispiel verweisen, auf den in Köln/CCAA bezeugten Titus Gesatius. Der Mann stammte wahrscheinlich aus der Sippe der Gesationes. Der Sippenname, der auf die im Raum Jülich/Iuliacum verehrten gesahenischen Matronen verweist, erscheint auf einem Weihestein aus Jülich/Iuliacum ${ }^{67}$ Für den Stein des Primio Cellissi fil. bietet sich ausgehend von den Überlegungen RÜGERS die Möglichkeit CVRIA GRVSDVAS als Personenname, als Name einer Dedikantin, zu interpretieren. Das Gentiliz Curius tritt allerdings vor allem in Italien auf. Die nächsten Belege stammen aus der Gallia Narbonensis und aus Noricum. ${ }^{6}$ Grusduas, das auf die aus Alt-Inden bekannten grusduahenischen Matronen verweist (s.o.), gesellt sich dagegen problemlos zu weiteren aus der Germania inferior bekannten femininen und maskulinen Personennamen auf -as, wie Rhenas, ${ }^{69}$ Tagamas $^{70}{ }^{\text {Valgas }}{ }^{71}$ oder Vihirmas. ${ }^{72}$

Mit der oben zitierten Wendung von FREMERSDORF lässt sich zur Zeit sicher behaupten, dass das Rätsel der dritten Zeile bisher noch nicht gelöst ist. Möglicherweise können weitere epigraphische Funde zu einer Klärung beitragen.

VIII.

In der Südmauer des Atriums von St. Gerion in Köln/CCAA fand sich 1977 ein Altarfragment (Höhe 32,5 cm - Breite 54,5 cm - Tiefe $23 \mathrm{~cm}$ ) aus Kalkstein. ${ }^{73}$ Fünf Jahre später entdeckte man ebenfalls im Atrium von St. Gerion - genauer im Fundament von Mauerabschnitt 82/15 - ein weiteres Altarfragment (Höhe $58 \mathrm{~cm}$ - Breite $42 \mathrm{~cm}-$ Tiefe $27 \mathrm{~cm}$ ) aus Kalkstein. ${ }^{74}$ Beide Fragmente mit Nebenseitenrelief wurden von B. und H. GALSTERER zunächst einzeln publiziert. ${ }^{75}$ Die Zusammengehörigkeit der

\footnotetext{
${ }^{64}$ RÜGER 1972, 256.

${ }^{65}$ Einen keltischen Genitiv Singular auf -as bzw. -ias bezeugt die Inschrift auf einem Fingerring aus Walheim [AE 1963, $120=4$. N. 477. Nr.37, Divixta Argentias (filia)]. Zur Kasusbildung s. W. SCHLEIERMACHER, Germania 40 (1962), 338. - In diesem Zusammenhang sei aufgrund der Endung nebenbei auf die bisher nicht eindeutig geklärte Herkunftsangabe DEVAS verwiesen: CIL XIII 6221 (Worms/Borbetomagus; Amandus Velugni f. Devas), 1. N. 6. Nr.20 (Trier/Augusta Treverorum; Optatius Verus Devas).

66 RÜGER 1987, 30.

67 4. N. 530. Nr.148 = AE 1967, $344=$ BILlER 2010, 77. f. - Zur Inschrift des Titus Gesatius s. AlFÖldy 1967, 5-8, A. KAKOSCHKE, FeRA 30 (2016), 13-15.

${ }^{68}$ Zum Namen s. ScHUlZE 1904, 286, OPEL II 88, KAKOSCHKE 2012, 91. GN 144.

${ }^{69}$ AE 2012, 976 (Köln/CCAA; Milia Rhenas).

${ }^{70}$ AE 2001, 1452 [Colijnsplaat/Ganuenta; Tagamas Tagadiani (filius)].

${ }^{71}$ CIL XIII $8307=$ IKöln² 298-299. Nr.359 [Köln/CCAA; Valgas Maieri (filius)].

72 CIL XIII 8771 [Ruimel; Fla(v)us Vihirmatis fil(ius)]. S. ferner je einen (fraglichen) Beleg für Proculas aus Floisdorf [CIL XIII 7898; C. Iul(ius) Proculas] und für Gracivas aus Enzen (CIL XIII $7901=$ LEHNER 1918, 312. Nr.779).

${ }^{73}$ Das Stück befindet sich heute im Römisch-Germanischen Museum in Köln (Inv.-Nr.77,94.14).

${ }^{74}$ Das Stück befindet sich heute im Römisch-Germanischen Museum in Köln (Inv.-Nr.82,115).

${ }^{75}$ IKöln $^{2}$ 131-132. Nr.132 = GALSTERER 1981, 236-237. Nr.7. Abb.7 (Photo) = AE 1981, 664, 208. Nr.244 = B. und H. GALSTERER, KJb 20 (1987), 98. Nr.23.
} 
Stücke (Abb.12) erkannte erstmals THOMAS, die den Stein (wie zuvor schon B. und H. GALSTERER die einzelnen Fragmente) in das Ende des zweiten Jahrhunderts bzw. in das beginnende dritte Jahrhundert $\mathrm{n}$. Chr. datiert. Für die unvollständige Inschrift des fragmentarischen Altars bietet THOMAS folgende Lesung an: ${ }^{76}$

$$
\begin{array}{ll} 
& \text { Matr[onis] } \\
\text { Aufan[iabus] } \\
\text { L(ucius) } ・ \text { Dom [itius] } \\
\text { Iri[---] } \\
\text { ex im }[\text { p(erio) ips(ius)] }
\end{array}
$$

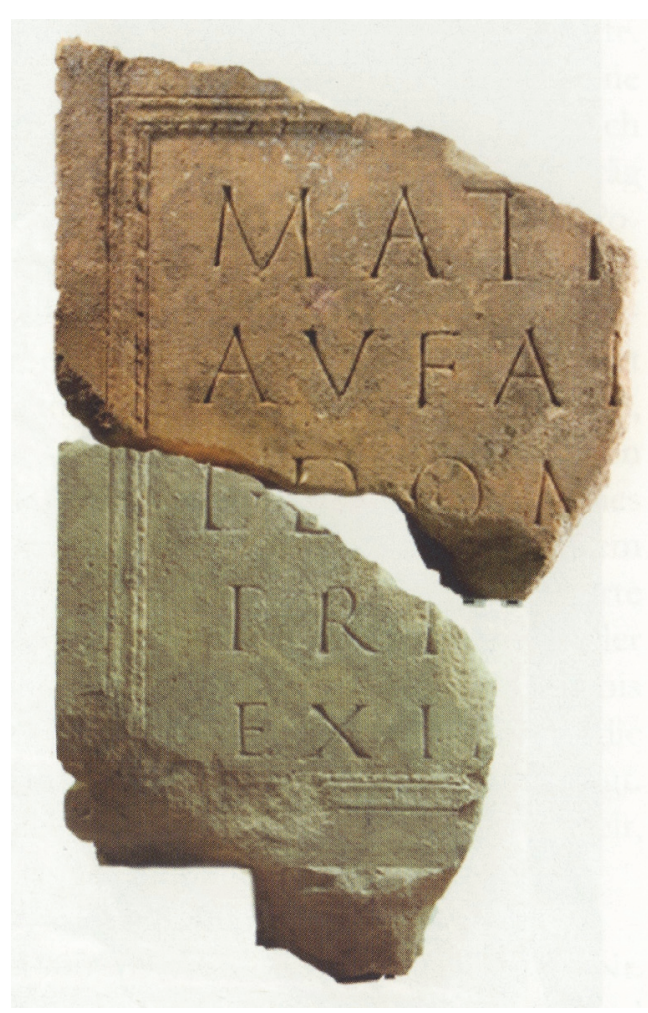

Abb.12: Fragmentarische Weihinschrift aus Köln/CCAA: (nach THOMAs 2014, 137-138. Nr.19. Abb.46)

Die Ergänzung der ersten drei Zeilen erscheint recht sicher. Für das mit DOM beginnende Gentiliz des Dedikanten kommt aufgrund fehlender Alternativen wohl nur der ausgeschriebene Name Domitius in Frage. ${ }^{77}$ Wie eine Rekonstruktionszeichnung (Abb.13) zeigt, hat der Steinmetz die ersten drei Zeilen offenbar gleichmäßig gefüllt. Gleiches gilt wohl auch für die letzten beiden Zeilen der Inschrift, die etwas eingerückt wurden. In der letzten Zeile kann mit einiger Sicherheit eine in Niedergermanien gängige Schlussformel wie ex imperio ipsarum libens merito oder ähnlich vermutet werden. Schwierigkeiten bereitet dagegen die Ergänzung und Lesung der vierten Zeile. ThOMAS liest hier B. und H. GALSTERER folgend die Buchstabengruppe IRI. B. und H. GALSTERER, die in einer älteren Publikation zunächst die Lesung FRA(!) boten, ${ }^{78}$ schreiben dazu: ,... in Z. 2 folgte dann wohl das Cognomen, wobei die

\footnotetext{
76 Thomas 2014, 137-138. Nr.19. Abb.44-46 (Photos).

77 Zum Namen s. KAKOSCHKE 2006, 163-164. GN 422. Zu weiteren seltenen mit DOM beginnenden Namen s. Solin/SALOMIES 1994, 69-70, OPEL II 104-106. Aus den germanischen Provinzen ist nur noch ein unsicherer Beleg für Domeius zu nennen (CIL XIII 6460, Meimsheim).

${ }^{78}$ B. und H. GALSTERER, KJb 20 (1987), 98. Nr.23.
} 
Lesung Tri- etwas weniger wahrscheinlich ist als Iri- (Irius als Namen Inscr. Ital. I, 1, 67 aus Salerno, vgl. auch CIL VIII 5005 und 7414 sowie ILALg II 5329/30).“ Wie von B. und H. GALSTERER angedeutet, bereitet die Suche nach einem Cognomen, das mit IRI beginnt erhebliche Probleme. ${ }^{79}$ Gleiches gilt bedingt im Übrigen auch für ein mit TRI oder ein mit FRI beginnendes Cognomen. ${ }^{80}$ Die Schwierigkeiten werden noch größer, nimmt man an, dass der Name wahrscheinlich die gesamte vierte Zeile der Inschrift füllte. Wenngleich grundsätzlich natürlich nicht ausgeschlossen werden darf, dass das Cognomen kürzer war und hinter dem Namen - sofern er recht kurz war - vielleicht noch eine stark abgekürzte Statusangabe stand, wie neg(otiator), vet(eranus) oder $m$ (issus) h(onesta) $m$ (issione). Für eine weitere Formel wie das in Niedergermanien beliebte pro se et suis reicht der Platz aber wohl auf keinen Fall aus.

Die noch vorhandenen Hasten bzw. Hastenreste am Anfang von Zeile 4 führen nach einem Vergleich mit dem überlieferten Namenmaterial der zwei germanischen Provinzen und der angrenzenden geographischen Räume mit hoher Wahrscheinlichkeit zu einem mit PRI beginnenden Namen, wie Primus, Privatus oder Priscus. Füllte der Name die Zeile ganz aus, kommen aufgrund der Länge der Zeile in erster Linie die Namen Primitivus, Primigenius (vielleicht mit einem Nexus) oder Priscianus in Frage.

Der onomastische Befund lässt sich mit dem epigraphischen möglicherweise in Deckung bringen. Bei dem letzten Buchstaben handelt es sich wohl, wie bereits von B. und H. GALSTERER richtig erkannt, um ein I, da alle anderen in Frage kommenden Buchstaben mit einer Vertikalen angesichts des vorangegangenen $\mathrm{R}$ oder aufgrund von nicht zu erkennenden Hastenresten bzw. nicht zu erkennenden Einkerbungen in der unvollständig vorhandenen Vertikalen auszuschließen sind. Auch ein M kommt wohl nicht in Frage, da der Steinmetz die seitlichen Vertikalen des Buchstabens recht schräg eingeschlagen hat. Der erste Buchstabe der Zeile, das vermeintliche I, steht sehr weit entfernt von dem folgenden R. Zwar ist auch der Abstand zwischen dem E und dem folgenden $\mathrm{X}$ zu Beginn der letzten Zeile recht groß, doch steht diese Lücke in keinem Verhältnis zu dem Abstand zwischen den Buchstaben am Beginn der vierten Zeile. Insofern scheint es überzeugend rechts der Vertikalen weitere Hastenreste zu vermuten. Entgegen B. und H. GALSTERER lässt sich die Vertikale jedoch nicht zu einem $\mathrm{T}$ oder F vervollständigen, sondern eher zu einem P. Die Reste einer geschwungenen Haste sind m.E. vor allem im oberen Bereich auch noch ansatzweise zu erkennen.

Der Text der fragmentarischen Inschrift aus Köln/CCAA könnte also wie folgt gelautet haben:

\author{
Matr[onis] \\ Aufan[iabus] \\ L(ucius) • Dom[itius] \\ Pri[---] \\ $5 \quad$ ex im[p(erio) ips(arum) l(ibens) m(erito)?]
}

\footnotetext{
${ }^{79}$ In Frage kommt z.B. Irinaeus, eine Variante des Namens Irenaeus. Der Name füllt die Zeile jedoch nicht ganz aus.

${ }^{80} \mathrm{Zu}$ möglichen Namen s. OPEL II 152, OPEL IV 129-130.
} 


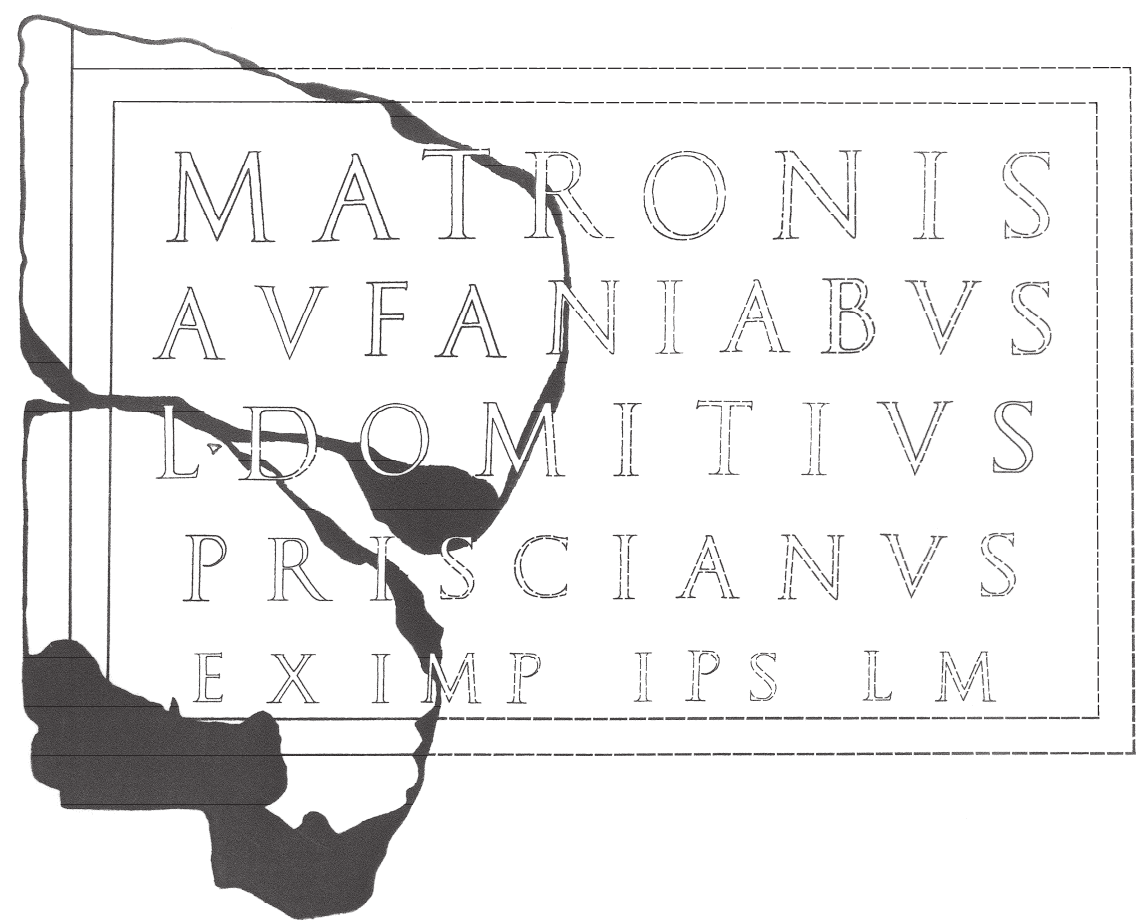

Abb.13: Leicht idealisierte Rekonstruktionszeichnung der Weihinschrift THOMAs 2014, 137-138. Nr.19 aus Köln/CCAA (Zeichnung A.K.)

Aufgrund der zahlreichen im Areal von St. Gereon entdeckten Matronensteine (39) lässt sich in der Umgebung der Kirche ein größeres Matronenheiligtum vermuten. THOMAS geht angesichts von neun in St. Gereon ausgegrabenen Weihungen an die aufanischen Matronen von einem Heiligtum für die aufanischen Matronen im näheren Bereich von St. Gereon aus. ${ }^{81}$ Für die Bedeutung dieses Heiligtums sprechen nicht nur die Fragmente eines ursprünglich möglicherweise $270 \mathrm{~cm}$ hohen Reliefs mit Matronendarstellung ${ }^{82}$ und ein Fragment, das wohl zu einem Weihestein mit einer Höhe von über $160 \mathrm{~cm}$ und einer Breite von ca. $120 \mathrm{~cm}$ gehört, ${ }^{83}$ sondern auch der hier vorliegende Weihestein. Trifft die vorgeschlagene Ergänzung zu, besaß der Stein des L. Dom[itius] Pri[---] mit ca. $115 \mathrm{~cm}$ (ohne Sockel) eine beachtliche Breite. Der Votivstein gesellt sich damit zu den größten Matronensteinen Niedergermaniens, die aus dem Matronenheiligtum aus Bonn/Bonna stammen. ${ }^{84}$

\footnotetext{
${ }^{81}$ THOMAS 2014, 102, 131. S. auch ECK/KOßMANN 2009, 81-82.

82 Thomas 2014, 98-101. Abb.2-3 (Photos), 162-166. Nr.92. Abb.77-79 (Photos). Zur vermuteten Größe des Reliefs s. H. GrEGAREK, KJb 37 (2004), 51-56. Abb.15 (Rekonstruktionszeichnung).

${ }^{83}$ ThOMAs 2014, 136-137. Nr.18. Abb.41-43 (Photos) = IKöln ${ }^{2}$ 134. Nr.137. Zur vermuteten Größe des Weihesteins s. P. NoELKE, BJb 190 (1990), 108-115. Nr.4. Abb.20-21 (Photos + Rekonstruktionszeichnung).

84 Eine Rangliste der größten Matronenaltäre aus Niedergermanien, bzw. aus Bonn/Bonna und Köln/CCAA, bieten ECK/KOßMANN 2009, 98-99. Die Liste wird angeführt vom Weihestein der Flavia Tiberina (2. N. 95. Nr.149) mit folgenden Maßen: Höhe $175 \mathrm{~cm}$ - Breite ca. $94 \mathrm{~cm}$.
} 


\section{Sigeln}

1. N.

2. N.

4. N.

$\mathrm{AE}$

$\mathrm{BJb}$

BRGK

CIL

ES

GFA

IKöln ${ }^{2}$

$\mathrm{KJb}$

OPEL I I - IV

$\mathrm{RE}$

WZ

ZPE

ALFÖLDY 1967

ALFÖLDY 1968
H. FINKE, Neue Inschriften, BRGK 17 (1927), 1-107, 198-231.

H. Nesselhauf, Neue Inschriften aus dem römischen Germanien und den angrenzenden Gebieten, BRGK 27 (1937), 51-134.

U. SCHILLINGER-HÄFELE, Vierter Nachtrag zu CIL XIII und zweiter Nachtrag zu FR. VOLLMER, Inscriptiones Baivariae Romanae. Inschriften aus dem deutschen Anteil der germanischen Provinzen und des Treverergebietes sowie Rätiens und Noricums, BRGK 58 (1977), 447-603.

L'annee épigraphique, Paris.

Bonner Jahrbuch, Bonn.

Bericht der Römisch-Germanischen Kommission, Mainz.

Corpus Inscriptionum Latinarum, Berlin 1863ff.

Epigraphische Studien, Köln-Bonn.

Göttinger Forum für Altertumswissenschaft, Göttingen. (www.gfa.gbv.de)

B. GALSTERer / H. GALSTERER, Die römischen Steininschriften aus Köln. IKöln ${ }^{2}$, Kölner Forschungen 10, Mainz 2010.

Kölner Jahrbuch für Vor- und Frühgeschichte, Köln.

Onomasticon Provinciarum Europae Latinarum $\mathrm{I}^{2}$-IV. Ex materia ab A. Mócsy, R. FELDMANN, E. MARTON et M. SZILÁGYI collecta, hrsg. von B. LÖRINCZ u.a., Budapest-Wien 1999-2005.

Paulys Real-Enzyklopädie der classischen Altertumswissenschaft, Stuttgart.

Westdeutsche Zeitschrift, Trier.

Zeitschrift für Papyrologie und Epigraphik, Bonn.

\section{Literaturverzeichnis}

G. ALFÖLDY, Epigraphisches aus dem Rheinland II, ES 4 (Sammelband), 1-43.

G. AlFöLDY, Die Hilfstruppen der römischen Provinz Germania inferior, ES 6, Düsseldorf. 
BEYER 1999/2000

BILLER 2010

DELAMARRE 2007

DONDIN-PAYRE 2012

ECK/KOßMANN 2009

GALSTERER 1975

GALSTERER 1981

GALSTERER 1983

GARMAN 2008

GUTENBRUNNER 1936

HARTLEY/DiCKINSON 2010

JUFER/LUGINBÜHL 2001
B. BEYER, Wer beschützte die Ubier? Römische Matronennamen und ihre Beziehung zu Ortsnamen. Jülicher Geschichtsblätter 67/68, 171-188.

F. BILLER, Kultische Zentren und Matronenverehrung in der südlichen Germania inferior, Osnabrücker Forschungen $\mathrm{zu}$ Altertum und Antike-Rezeption 13, Rahden/Westf.

X. Delamarre, Nomina Celtica antiqua selecta inscriptionum (Noms de personnes celtiques dans l'épigraphie classique), Paris.

M. Dondin-PAYRE, À côté des collèges: les curies des provinces nord-occidentales de l'empire romain, in: $\mathrm{M}$. Dondin-PAYre / N. Tran (Hrsg.), Collegia. Le phénomène associatif dans l'Occident romain, Paris, 81101.

W. ECK / D. KOßMANN, Votivaltäre in den Matronenheiligtümern in Niedergermanien: Ein Reflex der städtischen und ländlichen Gesellschaften einer römischen Provinzstadt, in: CH. AufFARTH (Hrsg.), Religion auf dem Lande. Entstehung und Veränderung von Sakrallandschaften unter römischer Herrschaft, Stuttgart, 73102.

B. GAlsterer / H. GAlsterer, Die römischen Steininschriften aus Köln, Wissenschaftliche Kataloge des Römisch-Germanischen Museums 2, Köln.

B. Galsterer / H. Galsterer, Neue Inschriften aus Köln - Funde der Jahre 1974-1979, ES 12 (Sammelband), 225-264.

B. Galsterer / H. Galsterer, Neue Inschriften aus Köln II - Funde der Jahre 1980-1982, ES 13 (Sammelband), 167-206.

A. G. GARMAn, The cult of the Matronae in the Roman Rhineland. An historical evalution of the archaeological evidence, Lewiston, NY.

S. GUTENBRUNNER, Die germanischen Götternamen der antiken Inschriften, Rheinische Beiträge und Hülfsbücher zur germanischen Philologie und Volkskunde 24, Halle (Saale).

B. R. Hartley / B. M. Dickinson, Names on Terra Sigillata. An Index of Makers' Stamps \& Signatures on Gallo-Roman Terra Sigillata (Samian Ware), Bd.6. MASCLUS I-BALBUS to OXITTUS, London.

N. Jufer / TH. LuginBÜHL, Les dieux gaulois. Répertoire des noms de divinités celtiques connus par l'épigraphie, les textes antiques et la Toponymie, Paris.

http://www.fera-journal.eu 
KAKOSCHKE 2006

KAKOSCHKE 2007

KAKOSCHKE 2008

KAKOSCHKE 2010

KAKOSCHKE 2012

KAKOSCHKE 2014

LEHNER 1915

LEHNER 1918

NEDOMA 2014

PÄFFGEN 1992

REUTER 2012

RAEPSAET-CHARLIER 1993

REICHERT 1987-90

RIESE 1914

ROTHENHÖFER 2014
A. KaKoschKe, Die Personennamen in den zwei germanischen Provinzen. Ein Katalog. Bd.1: Gentilnomina ABILIUS-VOLUSIUS, Rahden/Westf.

A. KAKoschKe, Die Personennamen in den zwei germanischen Provinzen. Ein Katalog. Bd.2,1: Cognomina ABAIUS-LYSIAS, Rahden/Westf.

A. KakoschKe, Die Personennamen in den zwei germanischen Provinzen. Ein Katalog. Bd.2,2: Cognomina MACCAUS-ZYASCELIS, Rahden/Westf.

A. Kakoschke, Die Personennamen in der römischen Provinz Gallia Belgica, Alpha - Omega. Reihe A. Lexika - Indizes - Konkordanzen zur klassischen Philologie 255, Hildesheim-Zürich-New York.

A. KakoschKe, Die Personennamen in der römischen Provinz Noricum, Alpha - Omega. Reihe A. Lexika Indizes - Konkordanzen zur klassischen Philologie 263, Hildesheim-Zürich-New York.

A. KAKOschKe, Hapax - Steinmetzirrtum - Überlieferungsfehler - Fehllesung - Fälschung. $\mathrm{Zu}$ einigen auffälligen Personennamen aus den zwei germanischen Provinzen, Stuttgart.

H. LEHNER, Führer durch das Provinzialmuseum in Bonn. Bd.1. Die antike Abteilung, Bonn.

H. LEHNER, Die antiken Steindenkmäler des Provinzialmuseums in Bonn, Veröffentlichungen des Provinzialmuseums in Bonn 9, Bonn.

R. Nedoma, Matronae Grusduahenae, Beiträge zur Namenforschung 49.4, 441-449.

B. PÄFFGEN, Die Ausgrabungen in St. Severin in Köln. 3 Bde., Kölner Forschungen 5,1-3, Mainz.

M. REUTER, Legio XXX Ulpia Victrix. Ihre Geschichte, ihre Soldaten, ihre Denkmäler, Xantener Berichte 23, Darmstadt-Mainz.

M.-Th. Raepsaet-Charlier, Diis Deabusque Omnibus. Formulaire votif et datation dans les Trois Gaules et les deux Germanies, Gallia Romana I, Paris.

H. REICHERT, Lexikon der altgermanischen Namen. 2 Teile, Thesaurus Palaeogermanicus 1, Wien.

A. RIESE, Das rheinische Germanien in den antiken Inschriften, Leipzig-Berlin.

P. ROTHENHÖFER, Fünf Weihinschriften aus dem römischen Rheinland, BJb 214, 27-42. 
RÜGER 1972

RÜGER 1987

SCHEID 2006

SCHÖNFELD 1911

SCHOPPA 1959

SCHULZE 1904

SIMEK 1984

SOLIN/SALOMIES 1994

SPAUL 1994

SPICKERMANN 1994

SPICKERMANN 2005
CH. B. RÜGER, Gallisch-Germanische Kurien, ES 9 (Sammelband), 251-260.

CH. B. RÜGER, Beobachtungen zu den epigraphischen Belegen der Muttergottheiten in den lateinischen Provinzendes Imperium Romanum, in: Matronen und verwandte Gottheiten. Ergebnisse eines Kolloquiums veranstaltet von der Göttinger Akademiekommission für die Altertumskunde Mittel- und Nordeuropas, Beihefte der Bonner Jahrbücher 44, Köln-Bonn, 1-30.

J. SCHEID, Les dévots en Germanie inférieure: divinités, lieux de culte, fidèles, in: M. DONDIN-PAYRE / M.-TH. RAEPSAET-CHARLIER (Hrsg.), Sanctuaires, pratiques cultuelles et territoires civiques dans l'Occident romain, Bruxelles, 297-346.

M. SCHÖNFELD, Wörterbuch der altgermanischen Personen- und Völkernamen, Germanische Bibliothek 1. Sammlung Germanischer Elementar- und Handwörterbücher 4. Reihe: Wörterbücher, Heidelberg. 1911.

H. SснорPA, Römische Götterdenkmäler in Köln, Die Denkmäler des römischen Köln 22), Köln.

W. Schulze, Zur Geschichte lateinischer Eigennamen, Abhdlg. der Königl. Ges. der Wiss. zu Göttingen. Phil.Hist. Kl. N. F. 5, Göttingen (Nachdr. Berlin-ZürichDublin 1966).

R. SIMEK, Lexikon der germanischen Mythologie, Stuttgart 1984.

H. Solin / O. SAlomies, Repertorium nominum gentilium et cognominum Latinorum. 2. Aufl. Editio nova addendis corrigendisque augmentata, Alpha Omega. Reihe A. Lexika - Indizes - Konkordanzen zur klassischen Philologie 80, Hildesheim-Zürich-New York.

J. E. H. SpAUL, $\mathrm{Ala}^{2}$. The auxiliary cavalry units of the pre-Diocletianic Imperial Roman army. A revision and updating of the article written by Conrad Cichorius and originally published in part 1 of Band 1 of Realencyclopädie der Classischen Altertumswissenschaften. 1893. Andover.

W. SPICKERMANN, „Mulieres ex Voto“. Untersuchungen zur Götterverehrung von Frauen im römischen Gallien, Germanien und Rätien (1.-3. Jahrhundert n. Chr.), Bochumer historische Studien. Alte Geschichte 12, Bochum.

W. SPICKERMANN, Keltische Götter in der Germania Inferior?, in: W. SPICKERMANN / R. WIEGELS (Hrsg.), 
SPICKERMANN 2008

THOMAS 2004

VENNEMANN 1993

WEISGERBER 1968

EDCS

$\mathrm{EDH}$

EDR

\section{Kontakt zum Autor:}

Dr. Andreas Kakoschke

Nelly-Sachs-Weg 1

D-49191 Belm

E-Mail: andreaskakoschke@hotmail.de Opladen.
Keltische Götter im Römischen Reich, Akten des 4. Internationalen Workshops „Fontes Epigraphici Religionis Celticae Antiquae" (F.E.R.C.AN.) vom 4.-6.10.2002 an der Universität Osnabrück, Möhnesee, 125-148.

W. SPICKERMANN, Germania inferior. Religionsgeschichte des römischen Germanien 2, Religion der Römischen Provinzen 3, Tübingen.

R. THOMAS, Denkmäler der Matronenverehrung in der CCAA (Köln), KJb 47, 91-178.

Th. Vennemann GeN. NieRfELD, Ein ubisches Lautgesetz, Beiträge zur Geschichte der deutschen Sprache und Literatur 115, 367-399.

J. L. Weisgerber, Die Namen der Ubier, Wissenschaftliche Abhandlungen der Arbeitsgemeinschaft für Forschung des Landes Nordrhein-Westfalen 34, Köln-

\section{Elektronische Datenbanken}

Epigraphik-Datenbank Clauss/Slaby (Frankfurt), Leitung: M. ClausS (Johann Wolfgang Goethe-Universität Frankfurt am Main), Stand: 01.10.2016 (www.manfredclauss.de).

Epigraphische Datenbank Heidelberg, Leitung: $\mathrm{CH}$. WITSCHEL (Ruprecht-Karls-Universität Heidelberg), Stand: 01.10.2016 (www.uni-heidelberg.de/institute/ sonst/adw/edh).

Epigraphic Database Roma, Leitung: S. PANCIERA, S. ORLANDI (Università di Roma - La Sapienza), Stand: 01.10.2016. (www.edr-edr.it). 